\title{
Cofactor Independent Phosphoglycerate Mutase of Brugia malayi Induces a Mixed Th1/Th2 Type Immune Response and Inhibits Larval Development in the Host
}

\author{
Prashant K. Singh, Susheela Kushwaha, Ajay K. Rana, and Shailja Misra-Bhattacharya \\ Division of Parasitology, CSIR-Central Drug Research Institute, B.S. 10/1, Sector 10, P.O. Box 173, \\ Jankipuram Extension, Sitapur Road, Lucknow, Uttar Pradesh 226031, India
}

Correspondence should be addressed to Shailja Misra-Bhattacharya; shailja_cdri@rediffmail.com

Received 23 February 2014; Revised 6 May 2014; Accepted 20 May 2014; Published 1 July 2014

Academic Editor: Amogh A. Sahasrabuddhe

Copyright (C) 2014 Prashant K. Singh et al. This is an open access article distributed under the Creative Commons Attribution License, which permits unrestricted use, distribution, and reproduction in any medium, provided the original work is properly cited.

Lymphatic filariasis is a major debilitating disease, endemic in 72 countries putting more than 1.39 billion people at risk and 120 million are already infected. Despite the significant progress in chemotherapeutic advancements, there is still need for other measures like development of an effective vaccine or discovery of novel drug targets. In this study, structural and immunological characterization of independent phosphoglycerate mutase of filarial parasite Brugia malayi was carried out. Protein was found to be expressed in all major parasite life stages and as an excretory secretory product of adult parasites. Bm-iPGM also reacted to all the categories of human bancroftian patient's sera including endemic normals. In vivo immunological behaviour of protein was determined in immunized BALB/c mice followed by prophylactic analysis in BALB/c mice and Mastomys coucha. Immunization with Bm-iPGM led to generation of a mixed Th1/Th2 type immune response offering $58.2 \%$ protection against larval challenge in $\mathrm{BALB} / \mathrm{c}$ and $65-68 \%$ protection in $M$. coucha. In vitro studies confirmed participation of anti-Bm-iPGM antibodies in killing of B. malayi infective larvae and microfilariae through ADCC mechanism. The present findings reveal potential immunoprotective nature of Bm-iPGM advocating its worth as an antifilarial vaccine candidate.

\section{Introduction}

Lymphatic filariasis (LF) is one of the oldest and most morbid and debilitating parasitic diseases [1] caused by three threadlike nematode worms, Wuchereria bancrofti, Brugia malayi, and B. timori. An estimated 130 million people in 72 countries are currently infected, and around 1.34 billion are at risk of infection $[2,3]$. Diethylcarbamazine (DEC), principally microfilaricidal, is the only drug of choice for the treatment of LF [4]. Mass drug administration (MDA) using DEC, ivermectin (IVN), and albendazole (ALB) combination has been quite successful; however, several rounds of MDA are required to reduce the level of infection to sustain transmission [5]. As no drug is available against adult parasite, alternative strategies like vaccine development need to be explored in order to improve the effectiveness of disease control and prevention programmes. With the availability of the draft genome sequence of $B$. malayi [6] and the sequencing of endosymbiont Wolbachia, mosquito vectors (Aedes and Anopheles), and the human host, the ability to carry out large-scale comparative genomics presents opportunities to understand the molecular basis of parasitism defining molecules and pathways unique to nematode development and parasitism that can be characterised as the novel antifilarial drug targets or vaccine candidates.

In the current study, molecular, biophysical, and immune characterisation of independent phosphoglycerate mutase (Bm-iPGM) of human filarial parasite B. malayi was carried out. Phosphoglycerate mutases, the key enzymes in the glycolytic and gluconeogenic pathways, exist in two different forms having different mechanism of action and structure and that are either cofactor (2,3-diphosphoglycerate) dependent or cofactor-independent. The independent form is predominant in plants, nematodes, bacteria, and archaea 
[7]. All experimentally characterised iPGMs from eubacteria, plants, and invertebrates are monomers with a molecular mass of $55-75 \mathrm{kDa}[8,9]$. The absence of iPGM from humans and being indispensable in all nematodes including the filariids [10] advocate its potential as anthelminthic drug target. Bm-iPGM was purified successfully using bacterial host E. coli. Circular dichroism (CD) and fluorescence spectra of the recombinant protein were obtained to determine its secondary structure and native conformation. The abundant sharing of Bm-iPGM by all the major life-forms of B. malayi and its release in the form of excretory-secretory products [11] pointed towards its immunogenic nature. In silico analysis of Bm-iPGM predicted it to be highly antigenic with the presence of both MHC I and MHC II binding peptides. The antigenic nature was further validated by the crossreactivity of Bm-iPGM with human bancroftian sera of different categories of LF which persuaded us to examine the immunoprophylactic efficacy of the recombinant protein in animal models of LF.

In vivo immune characterisation of $\mathrm{Bm}-\mathrm{iPGM}$ in $\mathrm{BALB} / \mathrm{c}$ mice revealed it to invoke a mixed type of Th1/Th2 immune response. The immunised animals (BALB/c and Mastomys) that were challenged with the infective larvae displayed reduced worm establishment. As active filarial infections are accompanied with downregulation of the host immune system, skewing the helper immune response of host to Th2 type, we propose that immunization with Bm-iPGM is capable of generating a mixed Th1/Th2 type response that is unfavourable for parasite establishment and was responsible for providing considerable protection against LF in mouse models thus validating Bm-iPGM to be possible vaccine candidate against LF.

\section{Materials and Methods}

2.1. Experimental Animals. Randomly bred, 6-8-week-old male BALB/c (35) and Mastomys coucha (36) were used in the experiment. The animals were maintained in proper housing condition at Laboratory Animal Facility at CSIRCentral Drug Research Institute (CDRI), Lucknow, India. Animals were fed on standard pellet diet and water ad libitum. The animals and the animal experimental procedures were approved by the Animal Ethics Committee of CDRI duly constituted under the provisions of CPCSEA (Committee for the Purpose of Control and Supervision on Experiments on Animals), Government of India. The study bears the IAEC number 83/09/Para/IAEC dated 27/04/09. All the experiments were performed in duplicate (both for Mastomys and BALB/c) and almost similar results were obtained in both the experiments and, therefore, pooled.

2.2. Parasites. Infective larvae (L3) of B. malayi were recovered from the laboratory bred vector mosquitoes (Aedes aegypti) fed on donor Mastomys $9 \pm 1$ day back [12]. L3 were isolated from gently crushed mosquitoes by Baermann technique, washed, and counted in Ringer's solution. Adult B. malayi worms and microfilariae (Mf) were collected from the peritoneal cavities of the infected jirds on day 80-85 after L3 inoculation.

2.3. Homology Modelling of Bm-iPGM and Amino Acid Sequences. The homology model for Bm-iPGM was generated using Phyre server [13]. Bm-iPGM structure was generated with $100 \%$ precision and $41 \%$ identity using structure of Bacillus anthracis cofactor-independent 2 phosphoglycerate mutase as template (PDB id: c2ifyA, length: $508 \mathrm{AA})$. The data generated was analysed by The PyMOL Molecular Graphics System, Version 1.3, Schrödinger, LLC, and the cartoon structure was generated. Amino acid sequence of Bm-iPGM was also aligned with iPGM from $B$. anthracis (accession number: 2IFY_A) using Clustal W programme.

2.4. In Silico Antigenicity Prediction. The antigenicity of Bm-iPGM was determined by Kolaskar and Tongaonkar method [14]. This semiempirical method predicts antigenic determinants based on the physicochemical properties of amino acid residues and the frequencies of their occurrence in experimentally known segmental epitopes. Prediction of immunodominant $\mathrm{T}$ cell antigenic sites from the primary sequence of Bm-iPGM was determined by ProPred-I and ProPred MHC class-II binding peptide prediction servers, which are online web tools for the prediction of peptide binding to MHC class-I (HLA-A1, HLA-A2, HLA-A0201, HLA-A0205, HLA-A1101, HLA-A3101, HLA-A3302, HLAB2102, HLA-A3501, HLA-A4403, and HLA-5101) and class II (HLA-DRB1_0101, HLA-DRB1_0301, HLA-DRB1_0401, HLADRB1_0701, and HLA-DRB1_0801) alleles [15, 16]. The highest ranking MHC I and MHC II binding peptides were highlighted in the cartoon structure of Bm-iPGM obtained earlier.

2.5. Cloning, Expression, and Purification of Bm-iPGM. Expression and purification of Bm-iPGM was done as described elsewhere with minor modifications [17]. Briefly, gene specific forward (5'AGTCGGATCCATGGCCGAAGCAAAGAATCG- $3^{\prime}$ ) and reverse (5'ATGCCTCGAGGGCTTCATTACCAATGGC3 ${ }^{\prime}$ ) primers having restriction sites for the enzymes BamHI (F) and XhoI (R) were synthesised. Amplification of gene was carried out using $1 \mu \mathrm{M}$ of each primer, $200 \mu \mathrm{M}$ of each dNTP (Fermentas, USA), 0.5 unit taq DNA polymerase (Invitrogen, USA), 1xPCR buffer, and $1.5 \mu \mathrm{M}$ of $\mathrm{MgCl}_{2}$ with the following reaction conditions: $95^{\circ} \mathrm{C}$ for $2 \mathrm{~min}$, followed by 29 cycles of $95^{\circ} \mathrm{C}$ for $1 \mathrm{~min}, 58^{\circ} \mathrm{C}$ for $1 \mathrm{~min}, 72^{\circ} \mathrm{C}$ for $2 \mathrm{~min}$, and 1 cycle at $72^{\circ} \mathrm{C}$ for $10 \mathrm{~min}$. The $1548 \mathrm{bp}$ amplified gene was cloned into pTZ57R/T (2886 bp) vector as per manufacturer's instructions (Fermentas, USA). Plasmid DNA was isolated, and the insert was verified by sequencing. The 1548 bp gene was subcloned into pET28a expression vector for protein expression and purification. The conditions like temperature, isopropyl $\beta$-D-thiogalactopyranoside (IPTG) concentration, and duration of induction were standardized for optimal expression of the protein in soluble form. Five $\mathrm{mL}$ cultures (E. coli strain BL21 (DE3)) were grown at $37^{\circ} \mathrm{C}$ in an incubator shaker at $220 \mathrm{rpm}$ and induced (at $\mathrm{OD}_{600}$ of $0.5-0.6$ ) for $4 \mathrm{~h}$ with $0.2,0.5$, and $1.0 \mathrm{mM}$ IPTG. After induction, cells 
were harvested by centrifugation at $7000 \mathrm{rpm}$ for $5 \mathrm{~min}$ and lysed in $5 \mathrm{~mL}$ sample buffer $(0.313 \mathrm{M}$ Tris- $\mathrm{HCl}, \mathrm{pH} 6.8,50 \%$ glycerol, $10 \%$ SDS, and $0.05 \%$ bromophenol blue) for analysis on 10\% SDS-PAGE (Laemmli 1970) along with uninduced vector control culture. To observe the solubility of recombinant protein, the cell pellet was resuspended in $1 \mathrm{~mL}$ of lysis buffer (50 mM Tris- $\mathrm{HCl}, \mathrm{pH}$ 7.5; $200 \mathrm{mM} \mathrm{NaCl}$; and $100 \mathrm{mM}$ DTT), sonicated at $10 \mathrm{db} / 10 \mathrm{~s}$ in a Soniprep 150 sonicator in cold. The cell lysate was centrifuged at $14,000 \mathrm{rpm}$ for 30 minutes to collect the supernatant containing soluble fraction and the remaining pellet containing insoluble fraction was resuspended in $1 \mathrm{~mL}$ of lysis buffer. Soluble and insoluble fractions were then analyzed in parallel on 10\% SDS-PAGE and visualized by Coomassie blue staining. For purification of the expressed recombinant protein, the supernatant was run on Ni-NTA agarose affinity column preequilibrated with $50 \mathrm{mM}$ Tris-HCl buffer ( $\mathrm{pH}$ 7.5) along with $200 \mathrm{mM} \mathrm{NaCl}$ and $10 \mathrm{mM}$ imidazole. The column was subsequently washed with the same buffer containing $25 \mathrm{mM}$ and $40 \mathrm{mM}$ imidazole and the recombinant protein was eluted with $250 \mathrm{mM}$ imidazole, analyzed on SDS-PAGE and protein expression was confirmed using anti-His antibody in Western blot. Following purification, the exact mass of Bm-iPGM was determined through matrix-assisted laser desorption/ionization time-offlight mass spectrometry (MALDI-TOF). The protein was dialysed in water $(\mathrm{O} / \mathrm{N})$ to remove any buffer content and diluted in 30\% acetonitrile (ACN) and $0.1 \%$ trifluoroacetic acid (TFA) solution in a matrix of sinapinic acid. Ten $\mu \mathrm{L}$ of the prepared sample was then pipetted on the plate and was air-dried to allow cocrystallization of the protein and the matrix; the target plate was loaded in the MALDITOF spectrometer (Applied Biosystems MDS Sciex 4800 Plus MALDI TOF/TOF, Foster City, CA, USA). To check whether the recombinant protein was biochemically active, the activity of recombinant Bm-iPGM was measured (data not shown) as described previously using a standard one-step enzyme-coupled assay [17].

2.6. Biophysical Investigations. CD measurements were made on JASCO J810 Spectropolarimeter calibrated with ammonium (+)-10-camphorsulfonate with $6 \mu \mathrm{M}$ protein in $10 \mathrm{mM}$ citrate, glycine, and HEPES (CGH) buffer of desired $\mathrm{pH}$ with a $2 \mathrm{~mm}$ path length cell at $25^{\circ} \mathrm{C}$ using the following parameters: $1 \mathrm{sec}$ response, $50 \mathrm{~nm} / \mathrm{min}$ scan speed, $0.1 \mathrm{~nm}$ data acquisition interval, 3 accumulations, and $2 \mathrm{~nm}$ bandwidth. The spectra were recorded over a range of $190-250 \mathrm{~nm}$. The values obtained were normalized by subtracting the baseline recorded for the buffer under similar conditions. The ellipticity was reported as molar ellipticity $(\theta)\left(\mathrm{mdeg} \cdot \mathrm{cm}^{-2} \cdot \mathrm{mol}^{-1}\right)$. Fluorescence spectra were recorded with Perkin Elmer LS50B luminescence spectrometer in a quartz cell of $5 \mathrm{~mm}$ path length. $6 \mu \mathrm{M}$ concentration of protein in $50 \mathrm{mM}$ phosphate buffer ( $\mathrm{pH} 7.0$ ) was incubated at $25^{\circ} \mathrm{C}$, before recording the spectra. Excitation wavelength was $280 \mathrm{~nm}$ and the spectra were recorded between $290 \mathrm{~nm}$ and $400 \mathrm{~nm}$.

2.7. Production of Polyclonal Antibodies to Analyze Stage Specific Expression of Bm-iPGM. Five BALB/c mice were administered subcutaneously with the recombinant BmiPGM $(25 \mu \mathrm{g} / \mathrm{animal})$ in three doses at 2 -week intervals. First dose was given in Freund's complete adjuvant (FCA), while the remaining two in Freund's incomplete adjuvant (FIA). The animals were euthanized a week after the last protein booster and blood was collected for serum separation. For preparation of soluble extracts, adult parasites, L3, and Mf were homogenized in sterile PBS ( $\mathrm{pH}$ 7.2) containing protease inhibiter cocktail (Sigma, USA) in cold and left for overnight $(\mathrm{O} / \mathrm{N})$ extraction at $4^{\circ} \mathrm{C}$ and were further sonicated and centrifuged. The protein content was estimated in the supernatant by Bradford method [18]. Protein was loaded on to $10 \%$ SDS-PAGE and transferred to nitrocellulose membrane (NC). Membrane strips were incubated with Bm-iPGM specific polyclonal antibody raised in mouse (1:5000). After washing, membranes were incubated with HRP-goat anti-mouse antibody and were developed with substrate o-phenylenediamine dihydrochloride (OPD) [19, 20]. Bm-iPGM gene expression in various stages of $B$. malayi was also observed using cDNA. Adult worms, L3, and Mf were recovered as mentioned above. RNA was extracted from all the three life stages using TRIzol reagent (Invitrogen, USA) and quantified with a GeneQuant (BioRad). After treatment with DNase I to eliminate genomic DNA contamination, $2 \mu \mathrm{g}$ of total RNAs from each life stage was used for the first cDNA synthesis using a first-strand cDNA synthesis kit (Invitrogen, USA). cDNAs were amplified with specific primer pairs under the conditions mentioned above.

2.8. Analysis of Bm-iPGM in Excretory and Secretory (ES) Product. Adult worms (4 worms $/ \mathrm{mL}$ ) were maintained in vitro in serum-free RPMI 1640 (GIBCO) supplemented with antibiotic antimycotic (Invitrogen, $100 \mathrm{U} / \mathrm{mL}$ penicillin, $100 \mathrm{mg} / \mathrm{mL}$ streptomycin, and $0.25 \mathrm{mg} / \mathrm{mL}$ of amphotericin B) and $25 \mathrm{mM}$ HEPES at $37^{\circ} \mathrm{C}$ in $5 \% \mathrm{CO}_{2}$ in air. The utilized media was collected and replaced with fresh medium every $24 \mathrm{~h}$ continuously up to 7 days. The medium collected was filtered through $0.2 \mathrm{mM}$ filters (Millipore, USA) and stored, pooled, and concentrated using $3 \mathrm{kDa}$ cut-off membranes filters (Millipore, USA). Concentrated ES product and recombinant $\mathrm{Bm}$-iPGM were individually run on $10 \%$ SDS-PAGE and transferred to NC membrane. Membrane was blocked with $3 \%$ skimmed milk for $1 \mathrm{~h}$ and incubated at room temperature (RT) with 1:200 dilution of anti-BmiPGM antibodies raised in $\mathrm{BALB} / \mathrm{c}$ mice. The membrane was reincubated with goat $1: 10,000$ dilution of anti-mouse IgG-HRP antibody for $1 \mathrm{~h}$ at $\mathrm{RT}$ and the reaction was developed with the substrate 3,3-diaminobenzidine (DAB) tetrahydrochloride.

\subsection{Reactivity of Bm-iPGM with Human Bancroftian Antibod-} ies. Reactivity of recombinant enzyme was observed with the antibody present in the sera of human subjects by Western blotting and ELISA. For serum, blood was collected from W. bancrofti endemic area in the outskirts of Lucknow, India, and was categorized as endemic normal, asymptomatic microfilaria carriers, microfilaraemic symptomatic, 
and amicrofilaraemic symptomatic. Sera from humans living in filaria free zones like Jammu and Kashmir, India, served as nonendemic control. Mf presence or absence was earlier determined in the $2 \mathrm{~mL}$ night blood by membrane filtration technique [21]. Purified recombinant protein along with prestained molecular weight marker was run on a preparative $10 \%$ SDS-PAGE, transferred to NC membrane, and processed for immune-recognition with human sera pools $(1: 200)$ of 10 subjects per category. Goat anti-human IgG-HRP (1:10,000 dilutions) was used as secondary antibody and reaction was developed by the DAB substrate.

IgG antibodies in individual sera sample (10 sera of each category) belonging to microfilaraemic, amicrofilaraemic symptomatic, endemic normal (EN), and nonendemic normal (NEN) categories were measured using recombinant BmiPGM as an antigen in ELISA as stated above. The human sera samples were added at 1:200 dilutions as primary antibody while goat anti-human IgG antibody-HRP $(1: 10,000)$ was used as secondary antibody.

\subsection{Immunization of $B A L B / c$ and Mastomys with Recom-} binant $B m$-iPGM. Immune characterization of recombinant $\mathrm{Bm}-\mathrm{iPGM}$ was carried out in $\mathrm{BALB} / \mathrm{c}$ while prophylactic efficacy was investigated both in BALB/c and in Mastomys. $\mathrm{BALB} / \mathrm{c}$ mice do not support full development of L3 to preadult or adult stage nor develop microfilaraemia while Mastomys being highly susceptible supports full development from L3 to adult with the release of Mf. Mice are ideal for immune characterization of an antigen. We divided the animals into three different treatment groups (ten BALB/c mice and twelve Mastomys per group were used) which received three equal immunization doses on day 0 , day 15, and day 23. Animals in treatment group 1 received only PBS (unimmunized control group), while animals in treatment group 2 received equivalent volume of FCA (day 15) and FIA (day 23) in PBS (adjuvant group). Animals in the last treatment group 3 were administered with $25 \mu \mathrm{g}$ recombinant protein along with the adjuvant (FCA on day 15 and with FIA on day 23), respectively. Preimmunized sera were collected from the retroorbital plexus of each mouse prior to immunization and thereafter on days 14 and 20 after first antigen dose. One week following final booster dose, half of the BALB/c from each group received 50 L3 of B. malayi each into the peritoneal cavity and were euthanized on day 15 after L3 challenge to assess the recovery of developing L3. The remaining 5 mice from each group were kept unchallenged and euthanized on day 30 post infection (p.i.) for investigating the immune responses generated by the recombinant protein.

Similarly Mastomys from all the three groups were challenged with $100 \mathrm{~L} 3$ of B. malayi subcutaneously (s.c.) one week after the final booster dose. Half of the animals from each group were euthanized on day 30 after L3 challenge and the remaining animals on day 180 after L3 challenge, respectively, to investigate the prophylactic efficacy and cellular proliferation in Bm-iPGM in immunized and control animals.
2.11. Bm-iPGM Specific Antibody and Isotype Levels in Sera by ELISA. IgG antibody titre and antibody isotypes were measured by ELISA. For measuring IgG antibody titre, the wells of ELISA plate (Nunc, Denmark) were coated with $1 \mu \mathrm{g} / \mathrm{mL}$ of Bm-iPGM $(100 \mu \mathrm{L} /$ well $)$ in carbonate buffer $\mathrm{pH} 9.6$ at $4^{\circ} \mathrm{C}$ overnight $(\mathrm{O} / \mathrm{N})$, blocked $(1 \%$ gelatin in PBS containing Tween-20) for 2 hours at $37^{\circ} \mathrm{C}$, and washed thrice with PBS-T with each single washing for $5 \mathrm{~min}$. Pooled serum of immunized and control group of animals (BALB/c) was used as primary antibody using serial twofold dilutions starting from 1:50 to 1:102400 while goat anti-mouse IgG-horse radish peroxidase (HRP) was added $(1: 10000)$ after washing and plate was incubated at $37^{\circ} \mathrm{C}$ for another $1 \mathrm{~h}$. Reaction was developed by adding OPD substrate prepared fresh $(20 \mathrm{mg}$ of OPD in $25 \mathrm{~mL}$ citrate buffer of $\mathrm{pH} 5.0$ and $20 \mu \mathrm{L}$ of $\mathrm{H}_{2} \mathrm{O}_{2}$ ) in dark for 10-15 minutes at RT and terminated by adding $2.5 \mathrm{~N} \mathrm{H}_{2} \mathrm{SO}_{4}$. Absorbance was read at $492 \mathrm{~nm}$ in an ELISA plate reader. For antibody isotyping, pooled sera $(\mathrm{BALB} / \mathrm{c}$; $1: 100)$ was used as primary antibody while goat-anti-mouse monoclonal antibodies to IgM, IgA, IgG1, IgG2a, IgG2b, and IgG3 $(1: 1000)$ and rabbit anti goat-IgG-HRP $(1: 5000)$ (Sigma antibody isotype kit, USA) served as secondary and tertiary antibodies, respectively. Reactions were measured after adding the substrate OPD as mentioned above. Mean of the triplicate OD values was calculated and was used for plotting the graph.

2.12. Oxidative Burst in Peritoneal Macrophages. Real-time monitoring of intracellular reactive oxygen species (ROS) in peritoneal exudate cells (PEC) of BALB/c was determined through a fluorometric assay using $2^{\prime}, 7^{\prime}$-dichlorofluorescein diacetate (DCF-DA) as described earlier [22] with minor modifications [20]. Briefly, freshly harvested PEC's (from immunized and control animals) at $1 \times 10^{6}$ cells/tube were probe loaded with the DCF-DA at final concentration of $1 \mu \mathrm{M}$ for $15 \mathrm{~min}$ at $37^{\circ} \mathrm{C}$ in $\mathrm{CO}_{2}$ incubator. ROS levels in individual living cells were determined by sequentially measuring their fluorescence intensity (FI) on FACSCalibur (BD, USA). Data was analyzed by CellQuest Software (BD, USA) and mean ROS values were evaluated for cell populations.

2.13. Immunophenotyping of $T$ and B Lymphocyte Population. Splenocytes from PBS/adjuvant control and Bm-iPGM immunized groups of BALB/c were used for immunophenotyping to assess lymphocyte subset population on a flow cytometer (FACSCalibur, BD, USA) using fluorochrome (FITC or PE) conjugated anti-mouse antibodies (Serotec, $\mathrm{UK}$ ) directed against receptors to CD4, CD8, and CD19 [21]. Splenocytes $\left(1 \times 10^{6}\right)$ were initially blocked with Mouse Seroblock FCR at RT for $10 \mathrm{~min}$, washed, and divided into different tubes for labelling with monoclonals to CD4+ and CD8+ T cells or CD 19+ B cells for $10 \mathrm{~min}$ at RT. Cells were washed and finally suspended in sheath fluid for analysis by FACSCalibur using CellQuest analysis software (BD, USA) after gating the forward and side-scatter settings to exclude debris. For each determination, 20,000 cells were analyzed and the results are reported as percentage of each cell population. 
2.14. Intracellular Th1 and Th2 Cytokine Response in Immunized $B A L B / c$. The measurement of intracellular cytokines in the spleen was done as per manufacturer's (BD, USA) protocol as mentioned earlier [21]. Briefly, splenocytes (4 $\left.\times 10^{6} / \mathrm{mL}\right)$ were incubated with brefeldin A $(10 \mu \mathrm{g} / \mathrm{mL})$ (Serotec, UK) in dark for $6 \mathrm{~h}$ at $37^{\circ} \mathrm{C}$ and reincubated with mouse Seroblock FcR at RT for another $10 \mathrm{~min}$. Cells were washed and incubated with FITC-rat anti-mouse CD4+ antibody. Leucoperm A and Leucoperm B (Serotec, UK) were added at RT for $15 \mathrm{~min}$ and cells were dispensed in four tubes each containing $1 \times 10^{6}$ cells $/ 100 \mu \mathrm{L}$. PE-rat anti-mouse monoclonal antibodies to cytokines interleukin- (IL-) 2, IL4, IL-10, and IFN- $\gamma$ were added to separate tubes and cells were finally suspended in $500 \mu \mathrm{L}$ of $0.5 \%$ paraformaldehyde for flow cytometer readings.

2.15. Cellular Immune Response in Immunized and Control Mastomys Groups. The proliferation of splenocytes isolated from the control and experimental Mastomys after vaccination and challenge was performed from both the batches as described earlier [23]. In brief, spleen was aseptically removed and cells were passed through a sterile nylon cell strainer ( $40 \mu \mathrm{m}$ pore size; BD Falcon, USA) to prepare single cell suspension. Cells $(100 \mu \mathrm{L} /$ well $)$ from the stock $\left(5 \times 10^{6}\right.$ cells $/ \mathrm{mL}$ ) were plated in a 96-well culture plate in triplicate and stimulated with $100 \mu \mathrm{L}$ Bm-iPGM (optimal concentration $2.5 \mu \mathrm{g} / \mathrm{mL})$ or concanavalin A $(2.5 \mu \mathrm{g} / \mathrm{mL}$; Sigma, USA) for $72 \mathrm{~h}$ and pulsed with $1.0 \mu \mathrm{Ci} /$ well of $[3 \mathrm{H}]$ thymidine $(3 \mathrm{H}-$ $\mathrm{Tdr}$, specific activity $18 \mathrm{Ci} / \mathrm{m}$ mole, BARC, India) for $18 \mathrm{~h}$ preceding harvest. The radioactive incorporation in cells was measured in a $\beta$-counter (Beckman Instruments, Palo Alto, CA) using scintillation fluid. The stimulation index (SI) was assessed as a ratio of mean cpm (counts per minute) values of stimulated and unstimulated cultures.

2.16. Effect of Bm-iPGM on Parasitaemia and Parasite Burden in $B A L B / c$ and Mastomys. The BALB/c mice were euthanized on day 15 after L3 challenge to observe effect of vaccination on development of L3 to L4. On the other hand, half of the Mastomys from all the three groups were euthanized on day 30 to assess effect of immunization on development of young adults and remaining half on day 180 after L3 challenge to monitor microfilaraemia as well as effect on the adult worm establishment [12]. Various tissues, namely, heart, lungs, testes, and lymph nodes, were isolated and teased gently in PBS to recover adult worms. Female worms were teased on glass slide in a drop of PBS and observed microscopically to observe the effect of protein on worm fecundity. Data were compared with that of controls and arithmetic means were calculated for blood Mf density, worm burden, and female worm reproductive potential.

2.17. In Vitro Antibody-Dependent Cellular Adhesion and Cytotoxicity. Adherence of PECs to the surface of Mf and L3 was observed as described earlier [20]. Mf (100) and L3 (10) were individually cocultured with $1 \times 10^{6}$ PECs isolated from normal Mastomys in 96-well plate in presence of serum collected from immunized and normal Mastomys.
Each well contained $100 \mu \mathrm{L}$ PECs, $50 \mu \mathrm{L}$ serum (1:32), and $25 \mu \mathrm{L}$ guinea pig serum as a source of complement. Plates were kept at $37^{\circ} \mathrm{C}$ in a $\mathrm{CO}_{2}$ incubator (Binder, Germany) and cell adherence on the surface of parasite and further cytotoxicity was noted microscopically after 1, 3, 6, 24, and $48 \mathrm{~h}$ of incubation. Cytotoxicity was expressed by considering the number of immobile or dead parasites by adherence of effector cells against the total number of parasites recovered within $48 \mathrm{~h}$. The percentage of cytotoxicity was calculated by subtracting the number of dead/immobile parasites from the total parasites taken, dividing the result by total number of parasites and finally multiplying by 100. Furthermore, the presence of Bm-iPGM antigen on the surface of Mf and L3 was also investigated by fluorescence microscopy using polyclonal antibody raised against Bm-iPGM. In brief, 10 L3/50 Mf were incubated with pooled serum (1:500 dilution) from Bm-iPGM immunized Mastomys (collected on day 30) for $4 \mathrm{~h}$ at $37^{\circ} \mathrm{C}$ in 48 -well flat-bottom tissue culture plates. The parasites were washed and reincubated with secondary antibody (goat anti-mouse IgG-FITC, 1:10000) for $2 \mathrm{~h}$ at RT on a rotor-shaker and parasites were finally transferred to glass slide for fluorescence microscopy (Nikon, Japan).

\section{Statistical Analysis}

Data were analyzed using one-way analysis of variance (ANOVA). Individual comparisons following ANOVA were made using the Newman-Keuls method with the help of statistical software PRISM 3.0. Results of flow cytometry and worm recovery have been presented as mean \pm S.E. The criterion for statistical significance between the results of immunized and control groups were as follows: $P<0.05$ was considered as significant, $P<0.01$ was considered as highly significant, $P<0.001$ was considered as very highly significant, and $P>0.05$ was considered as nonsignificant.

\section{Results}

4.1. Bm-iPGM is Composed of Two Identical Domains and Is Highly Antigenic. Amino acid sequence alignment of Bm-iPGM with iPGM from $B$. anthracis using Clustal W showed 41\% identity (Figure 1(a)). Bm-iPGM structure was generated with $100 \%$ precision and $41 \%$ identity using the same structure of $B$. anthracis cofactor-independent 2 phosphoglycerate mutase taken as template (PDB id: c2ifyA, length: 508 AA). The data analysed by "The PyMOL Molecular Graphics System" showed Bm-iPGM to be composed of two identical domains connected by two linkers. Both the domains show similar folds containing central $\beta$ sheet structure which are flanked on both sides by $\alpha$ helices (Figure 1(b)). The in silico prediction showed 21 antigenic determinants in the protein with an average propensity being 1.0233 (see Supplementary Table 1 available online at http://dx.doi.org/10.1155/2014/590281). A number of MHC binding peptides were identified for the alleles used in analysis and Table 1 shows the best predicted binding peptide for each allele used in analysis and their log score. These 


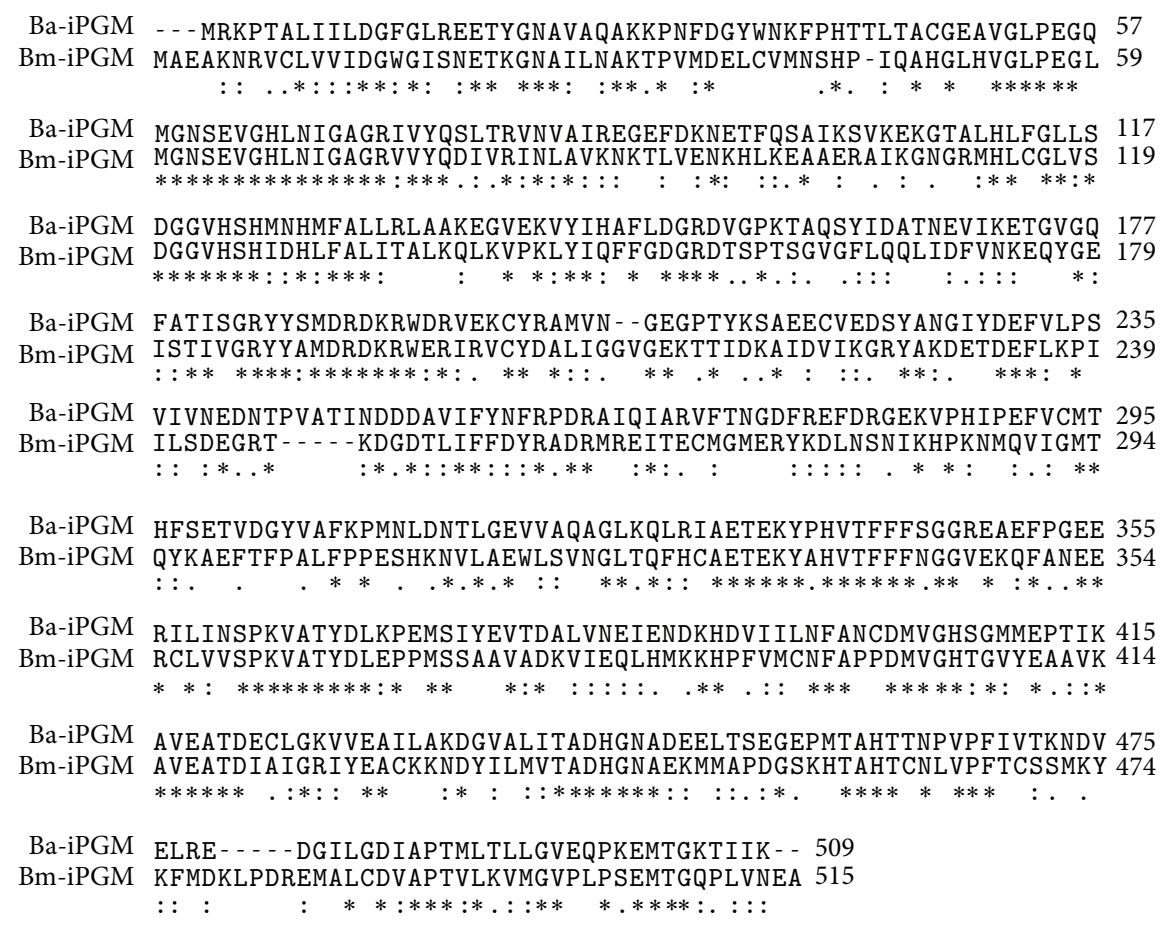

(a)

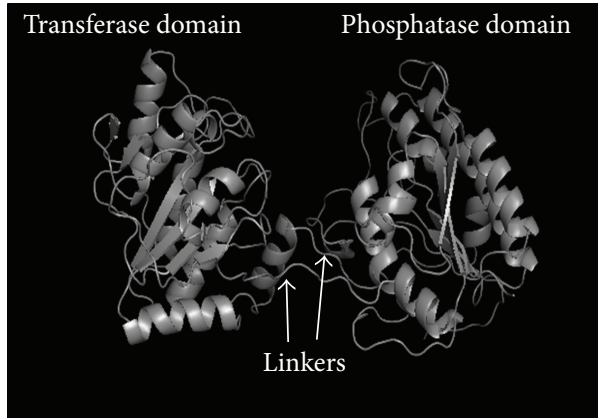

(b)

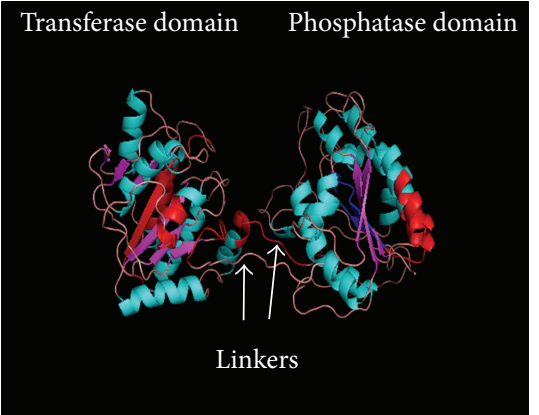

(c)

FIgURE 1: Amino acid sequence alignment and in silico structure of Bm-iPGM. (a) Multiple sequence alignment of the deduced amino acid sequence of Bm-iPGM; Bm-iPGM sequence was aligned with Bacillus anthracis cofactor-independent 2 phosphoglycerate mutase taken as template (Accession no. 2IFY_A) using Clustal W. Bm-iPGM showed 41\% identity with amino acid sequence of B. anthracis iPGM. Regions of identity (*), strong similarity (:), and weak similarity (.) are displayed. (b) In silico cartoon structure of Bm-iPGM. Helical content matches with the experimental CD data. (c) Bm-iPGM in silico cartoon structure showing quantitatively predicted MHCI and MHCII binding stretches (regions in red are presented by MHCI while the dark blue are presented by MHCII).

peptides were also visualised and highlighted in the cartoon structure of Bm-iPGM (Figure 1(c)).

\subsection{Bm-iPGM Was Cloned, Recombinant Protein Optimally} Expressed as a Single Band of $\sim 60 \mathrm{kDa}$. The $1548 \mathrm{bp}$ gene was successfully cloned into pTZ57R/T (2886 bp) vector, gene sequence verified by sequencing, and subcloned into expression vector pET 28a. The maximal protein expression was obtained after four hours of $0.5 \mathrm{mM}$ IPTG induction at $37^{\circ} \mathrm{C}$. A $\sim 60 \mathrm{kDa}$ recombinant protein band authenticated the expressed protein to be recombinant protein in-frame with the N-terminal 6x-His Tag (Figures 2(a) and 2(b)) which was found to be biochemically active. The exact mass of recombinant protein was found to be $61.779 \mathrm{kDa}$ as analyzed by MALDI-TOF (Figure 2(c)).

4.3. Secondary Structure Analysis by Spectroscopy Revealed $B m$-iPGM to Be $\alpha / \beta$ Type Protein. Far-UV CD spectrum can be used empirically as "blueprint" of a particular protein, providing information about the polypeptide backbone and the protein conformation in terms of its secondary structure [24]. The secondary structure of Bm-iPGM as characterized by far-UV CD shows that it is $\alpha / \beta$ type protein. As depicted in Figure 2(d), Bm-iPGM has two negative peaks around $222 \mathrm{~nm}$ and $208 \mathrm{~nm}$ and a stronger positive peak near $190 \mathrm{~nm}$, which is a characteristic of predominant $\alpha$-helical protein 


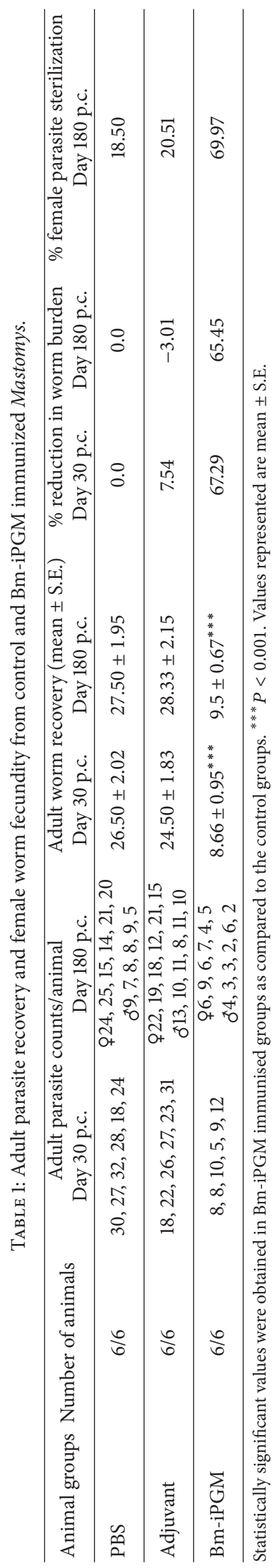




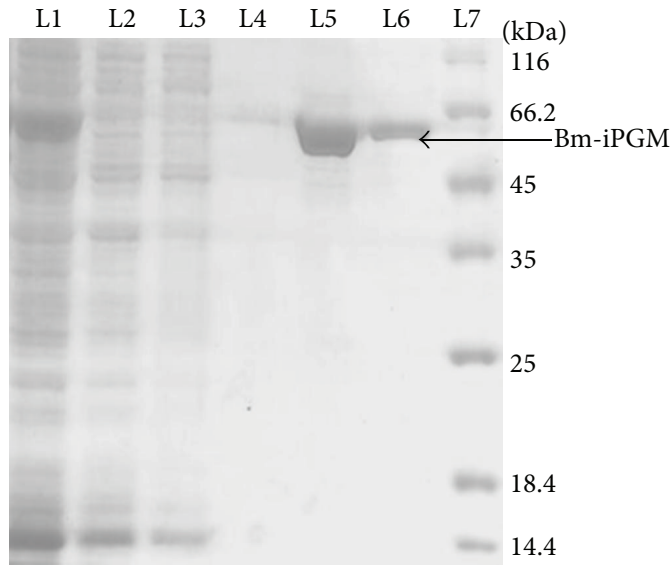

(a)

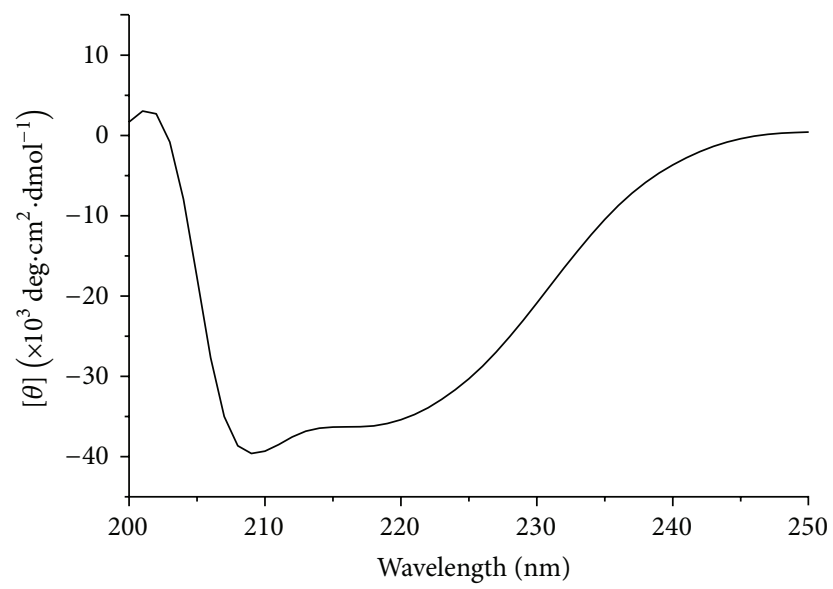

(d)

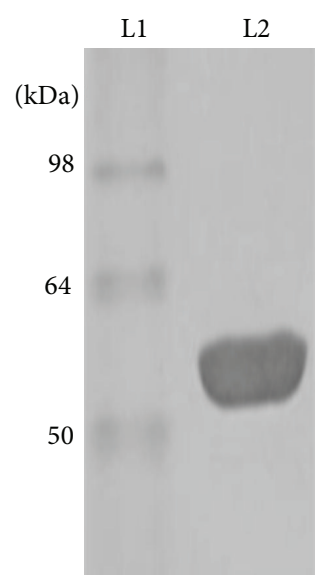

(b)

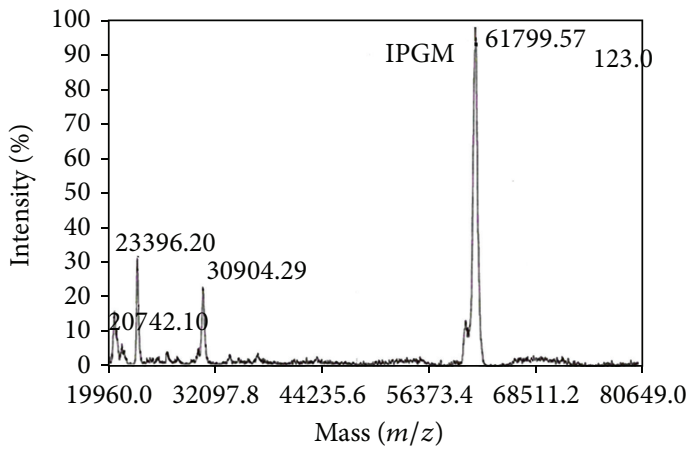

(c)

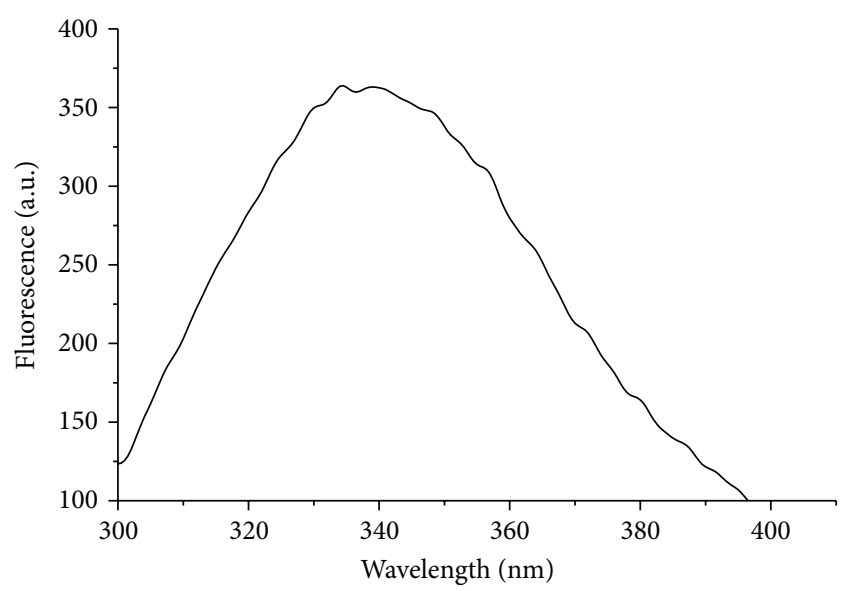

(e)

Figure 2: Cloning, expression, and purification of Bm-iPGM. (a) Purification of Bm-iPGM: L1: flow through; L2-L4: wash 1-3; L5 and L6: elute 1-2; L7: standard protein marker $(\mathrm{kDa})$. (b) Western blot analysis using anti-His mAb: L1: prestained protein marker; L2: purified Bm-iPGM. (c) MALDI-TOF analysis of the molecular mass of recombinant Bm-iPGM. A single major peak confirmed the mass of recombinant BmiPGM to be $61.799 \mathrm{kDa}$. (d) Far-UV CD spectra of Bm-iPGM; CD measurements were made on JASCO J810 spectropolarimeter calibrated with ammonium (+)-10-camphorsulfonate with $6 \mu \mathrm{M}$ protein in $10 \mathrm{mM}$ CGH buffer. (e) Fluorescence emission spectra of Bm-iPGM and spectra of Bm-iPGM in $50 \mathrm{mM}$ phosphate buffer were recorded with Perkin Elmer LS50B luminescence spectrometer. On excitation at $280 \mathrm{~nm}$, maximum emission spectra were noted at $340 \mathrm{~nm}$.

secondary structure [25]. Analysis of the averaged far-UV CD spectrum gave an estimate of $56.26 \% \alpha$-helix and $5.69 \% \beta$ strands. The intrinsic fluorescence of Bm-iPGM was studied to disclose the microenvironment surrounding the residues of tyrosine and tryptophan. When excited at $280 \mathrm{~nm}$, the maximum emission of Bm-iPGM was recorded at $340 \mathrm{~nm}$ (Figure 2(e)) revealing that tyr and trp residues were mainly located in hydrophobic environment and the Bm-iPGM was purified in its native form.

4.4. Bm-iPGM Is Expressed by All the Major Life-Forms of B. malayi and Is Excreted Out by Adult Worms. The polyclonal antibodies raised against the recombinant Bm-iPGM reacted with the native protein in lysates of adult parasites, Mf, and L3 (Figure 3(a)). Bm-iPGM gene was also amplified from cDNA of three major life stages of B. malayi using gene specific primers (Figure 3(b)). The results demonstrate presence of Bm-iPGM in all the three life stages analysed, thus demonstrating it to be an abundant protein. Polyclonal antibodies raised against Bm-iPGM reacted with recombinant $\mathrm{Bm}$-iPGM and with the ES product demonstrating it to be present in the in vitro excretory-secretory products of female B. malayi worms (Figure 3(c)).

4.5. Human W. bancrofti Patients Harbour Serum IgG Antibodies to Bm-iPGM. The recombinant protein showed good immunoreactivity in Western blot with bancroftian human sera belonging to different clinical categories, namely, nonendemic normals (NEN), endemic normals (EN), asymptomatic microfilaraemic $(\mathrm{Mf}+\mathrm{ve})$ carrier, microfilaraemic 


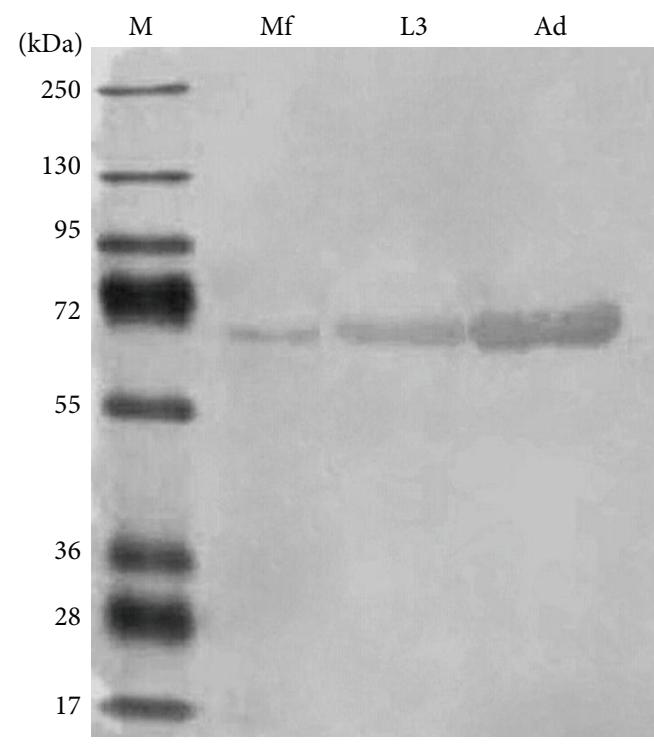

(a)

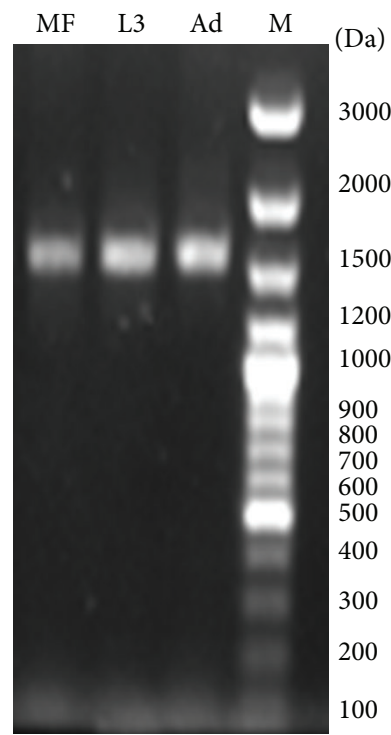

(b)

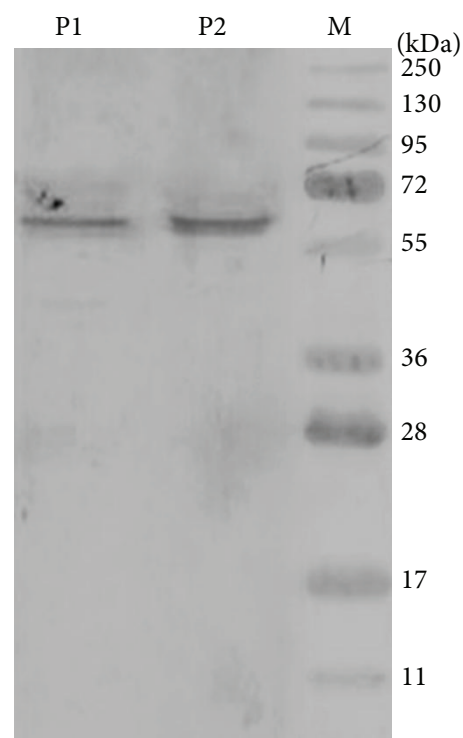

(c)

FIGURE 3: Stage specific expression of Bm-iPGM. (a) Western blot displaying expression of Bm-iPGM. M: standard protein marker; Mf: microfilariae, L3: infective larvae, and Ad: adult parasites. (b) 1.0\% agarose gel displaying Bm-iPGM amplification; Bm-iPGM gene was amplified from cDNA of three major life stages of B. malayi using specific primers. Mf: microfilariae, L3: infective larvae, Ad: adult parasites, and M: standard DNA marker. (c) Bm-iPGM in excretory-secretory products of adult parasite. Western blot was done to confirm presence/absence of iPGM enzyme in the ES product of B. malayi. Anti-Bm-iPGM antibody raised in mouse showed reactivity with the purified recombinant protein as well as the ES product of adult parasite. P1: purified recombinant Bm-iPGM, P2: adult worm ES product.

symptomatic (MFC), and amicrofilaraemic symptomatic (AMFCS), demonstrating the presence of Bm-iPGMin the target human parasite W. bancrofti (Figure 4(a)). The individuals from NEN category who are not exposed to filarial larvae did not display any reactivity with the recombinant enzyme showing filarial specificity of the expressed protein.

Bm-iPGM specific IgG ELISA was also carried out to determine the seroreactivity of individual category of serum samples from microfilaraemic, amicrofilaraemic symptomatic, EN, and NEN individuals. All the former three groups analysed revealed elevated levels of anti-Bm-iPGM IgG antibody with amicrofilaraemic symptomatic patients displaying highest antibody titre which was significant over the other groups $(P<0.01)$ (Figure $4(\mathrm{~b}))$. However nonendemic normals did not react to Bm-iPGM.

4.6. Bm-iPGM Generates Vigorous Antibody Response in $B A L B / c$ Mice. Antibody levels were measured in the sera obtained when the animals were euthanized on day 30 post infection (p.i.). The Bm-iPGM group developed higher levels of Bm-iPGM specific antibodies compared to PBS control and FCA/FIA group. Anti-Bm-iPGM antibody level remained higher in Bm-iPGM group even at $1: 6400$ dilution. None of the controls developed Bm-iPGM specific antibody response (Figure 5(a)). Measurement of Bm-iPGM specific IgG isotypes in experimental groups revealed that animals immunized with recombinant Bm-iPGM induced predominantly elevated level of IgG1, IgG2a, IgG2b, IgG3, IgM, and IgA (Figure 5(b)). IgG2a/IgG1 ratio was indicative of a mixed type of Th1/Th2 immune response. Animals from control groups did not develop Bm-iPGM specific antibody isotypes.

4.7. Bm-iPGM Activates the Antigen Presenting Cells (APCs) Upregulating the Production of Reactive Oxygen Species. A real time monitoring of oxidative burst generated from peritoneal macrophages of immunized and controls group of $\mathrm{BALB} / \mathrm{c}$ was done. Flow cytometry data indicate that BmiPGM immunization led to the generation of significantly higher oxidative burst $(P<0.01)$ in macrophages from $\mathrm{Bm}$ iPGM group as compared to the controls which might have played important role in parasite death (Figures 6(a) and 6(b)).

4.8. Increased Number of Both T And B Cell Population Was Observed. Bm-iPGM administration into $\mathrm{BALB} / \mathrm{c}$ mice led to expansion of both cellular and humoral immune response and a significant rise in CD4+ $(P<0.001)$ and CD8+ $(P<$ $0.01)$ T cells (Figures 6(c) and 6(d)). CD 19+ B cell population also increased significantly $(P<0.05)$ (Figure 6(e)). The PBS and FCA/FIA control groups of animals did not showed such heightened cellular and humoral immune response. Experiments were carried out to illustrate the in vitro proliferation of splenocytes from both the batches of Mastomys euthanized on day 30 and day 180 after larval challenge in presence of Bm-iPGM or mitogen Con A. Spleen cells from BmiPGM immunized animals exhibited noticeable proliferation whether stimulated with Con A or Bm-iPGM at both time points (Figures 7(a) and 7(b)). 


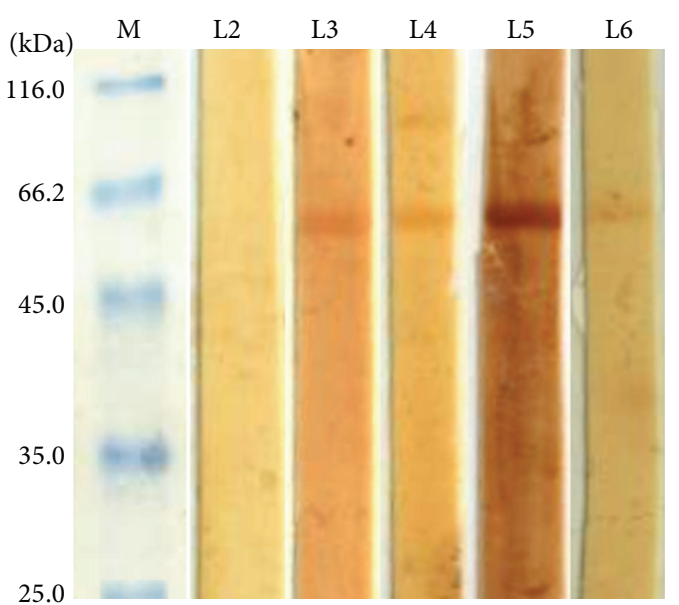

(a)

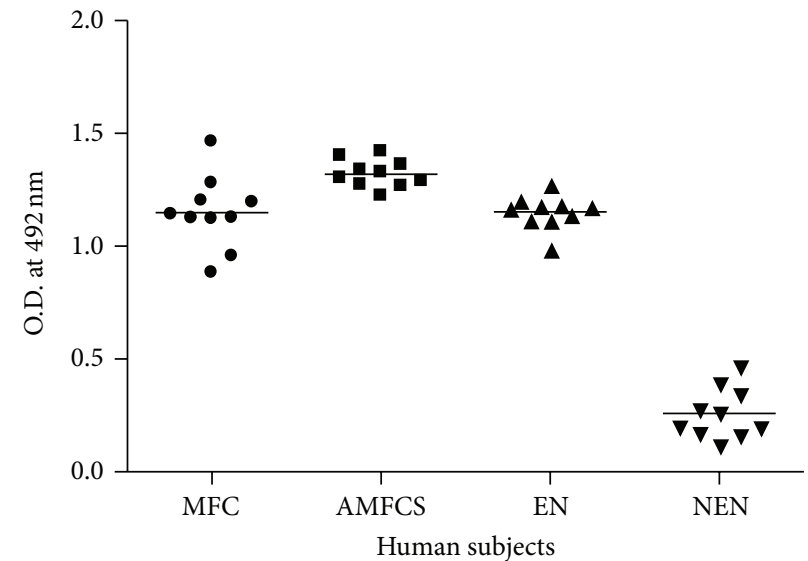

(b)

FIGURE 4: Seroreactivity of Bm-iPGM with human bancroftian sera pooled from 10 filarial patients of each category. (a) Western blots showing cross-reactivity of recombinant Bm-iPGM with various categories of human bancroftian sera. L1: standard protein marker; L2: nonendemic normal; L3: endemic normal; L4: microfilaraemic asymptomatic; L5: microfilaraemic symptomatic; L6 amicrofilaraemic symptomatic patients. (b) Bm-iPGM specific ELISA showing reactivity of Bm-iPGM with human bancroftian sera taken from human subjects belonging to various categories; MFC: microfilaraemic carrier, AMFCS: amicrofilaraemic symptomatic, EN: endemic normal, and NEN: nonendemic normal individuals. Serum $(1: 200)$ from ten individuals per clinical category was tested in ELISA for reactivity with recombinant Bm-iPGM. All the three groups from filarial endemic area revealed elevated levels of anti-Bm-iPGM antibodies.

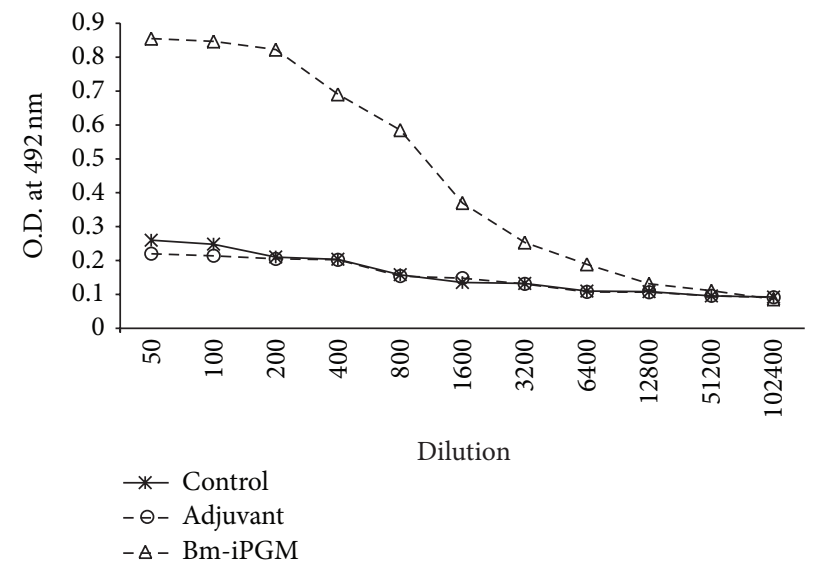

(a)

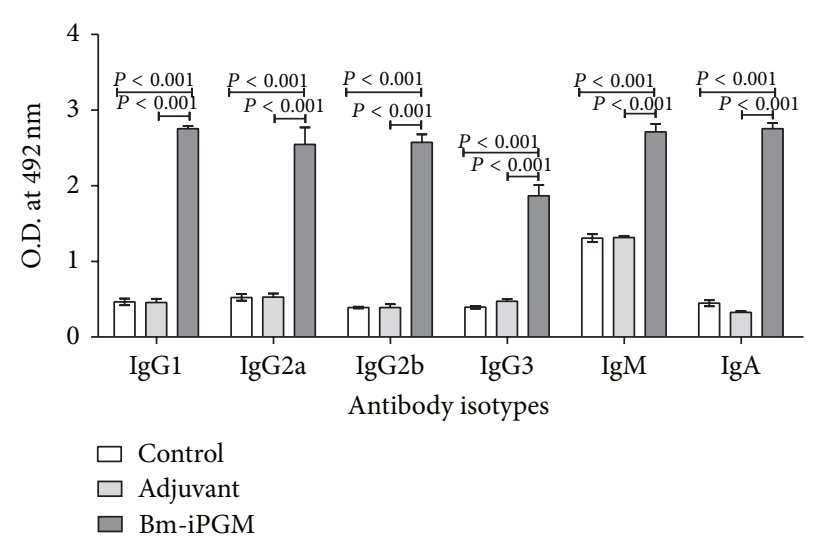

(b)

FIGURE 5: Bm-iPGM specific IgG antibody and antibody isotypes. (a) Antibodies were detected by ELISA in the pooled sera of BALB/c mice administered with Bm-iPGM along with adjuvant and PBS only. Elevated IgG level was maintained in the Bm-iPGM immunized group. (b) Anti-Bm-iPGM antibody isotype levels (IgG1, IgG2a, IgG2b, IgG3, IgM, and IgA) in the pooled sera of Bm-iPGM immunized, adjuvant immunized, and control groups. Considerable increase in the levels of all the isotypes was noticed. Each bar represents mean of triplicate OD values taken at $492 \mathrm{~nm}$ each obtained with pooled sera of five experimental animals.

4.9. Immunization with Bm-iPGM Elicits a Mixed Th1/Th2 Immune Response with Marked Reduction in Larval Development in BALB/c While in Mastomys Considerably Reduced Microfilarial Density, Adult Worm Recovery, and Female Worm Fecundity Were Observed. The levels of both proinflammatory and anti-inflammatory cytokines were determined intracellularly in the splenic cell population of immunized $\mathrm{BALB} / \mathrm{c}$ mice. There was an up regulation in the levels of proinflammatory cytokines IL-2 $(P<0.001)$ and IFN- $\gamma$ $(P<0.01)$ as well as anti-inflammatory cytokines IL-4 $(P<$ $0.01)$ and IL-10 $(P<0.01)$ (Figures $8(\mathrm{a}), 8(\mathrm{~b}), 8(\mathrm{c})$, and $8(\mathrm{~d}))$ as analysed by flow cytometry which indicated generation of a mixed Th1/Th2 immune response. Immunization of BALB/c mice with Bm-iPGM resulted in a significant reduction in worm establishment in Bm-iPGM (number of parasites $8.6 \pm$ 1.1) immunized animals $(P<0.001)$ as compared to the PBS 


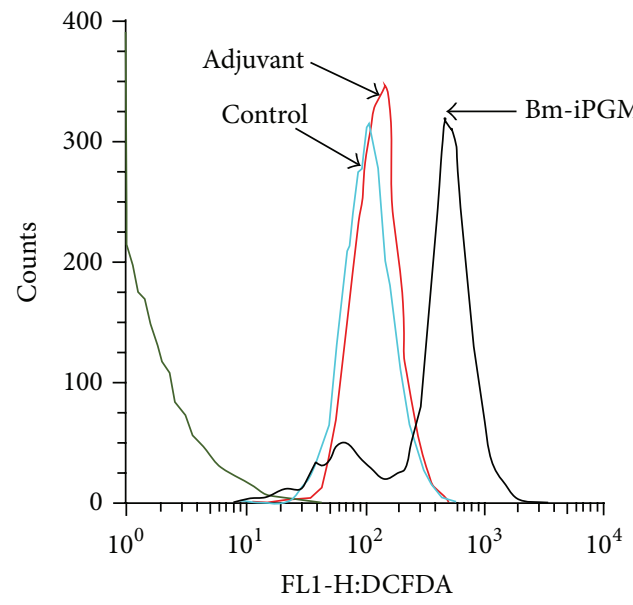

(a)

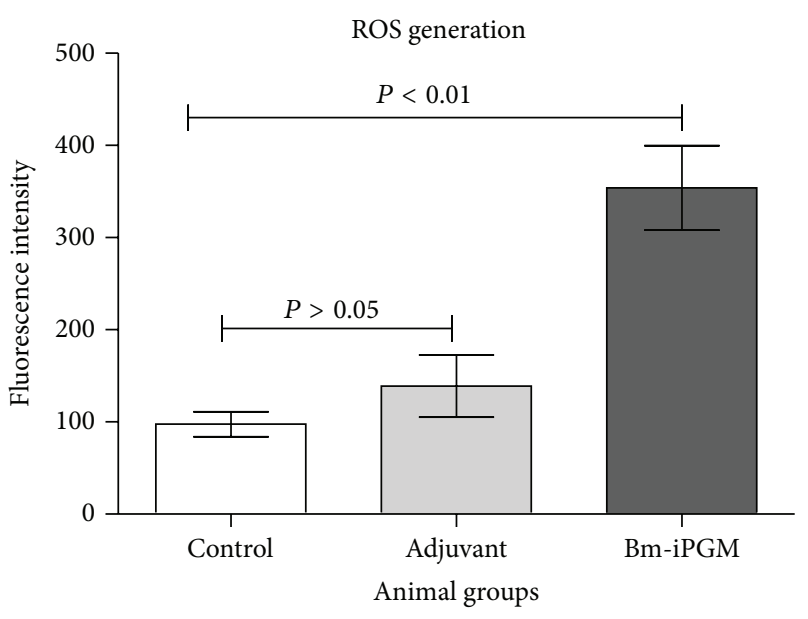

(b)

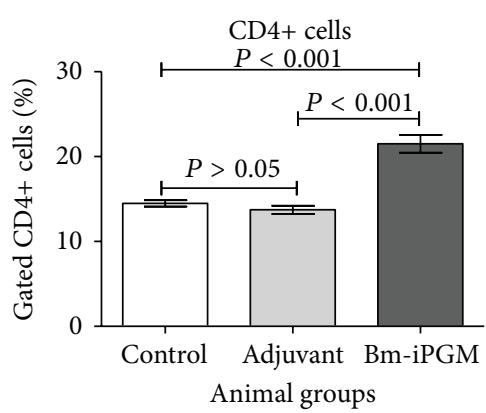

(c)

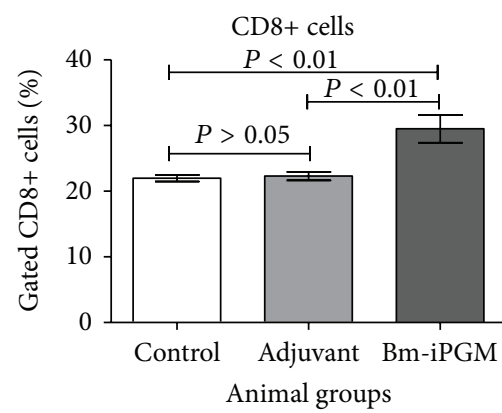

(d)

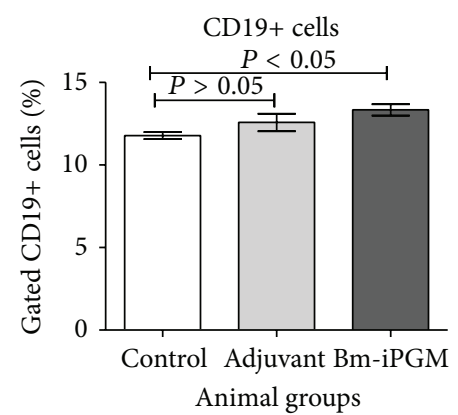

(e)

FIGURE 6: Flow cytometric analysis of ROS generation by peritoneal macrophages and splenic lymphocyte surface staining for T and B cells. (a) Representative FACS histogram for ROS generation from each group is displayed and values closer to mean are represented. (b) Reactive oxygen species produced by the peritoneal macrophages $\left(1 \times 10^{6} / \mathrm{mL}\right)$ isolated from for all groups were loaded with probe DCF-DA and ROS generation was evaluated on day 30 when the animals were euthanized. Bm-iPGM immunization led to activation of macrophages which significantly generated reactive oxygen species $(P<0.01)$. (c) CD4+ T cell marker, (d) CD8+ T cell marker, and (e) CD19+ B cell marker. Significant increase was noticed in the number of CD4+ T cells $(P<0.001)$ and CD8+ T cells $(P<0.01)$ from Bm-iPGM immunized animals. Though a marginal expansion in B cell population was observed, it was statistically significant $(P<0.05)$.

control (number of parasites $20.60 \pm 1.2)$ and FCA/FIA $(22.40$ \pm 1.43 ) groups. Thus, immunization with Bm-iPGM resulted in up to $58.25 \%$ reduction in parasite establishment when $\mathrm{BALB} / \mathrm{c}$ mice were euthanized on day 15 p.c. (Figure 9(a)).

Immunization of Mastomys with Bm-iPGM had profound adverse effect on the Mf density and adult worm establishment when compared with the nonimmunized controls. Though Mf appeared in all the three groups by day 90, (Figure 9(b)) their density was much lower $(108 \pm 25.34)$ in Bm-iPGM immunized group in contrast to $391.3 \pm 77.32$ and $293 \pm 100.6$ in PBS and adjuvant groups, respectively, at the time of euthanization demonstrating $72.4 \%$ reduction over that of control $(P<0.05)$. Considerable reduction (65.45-67.29\%) in adult worm recovery was noticed in BmiPGM immunized groups when the animals were euthanized on days 30 and 180 after larval challenge (Table 1). In contrast to immunized groups ( $8-10$ worms/animal), the average recovery of adult worms ranged between 24.50 and 28.0 in the two control groups. Vaccination also led to significant adverse effect on the female worm fecundity as observed on day 180
(Table 1). The percentage of sterile adult females recovered from Bm-iPGM gp was $69.97 \pm 4.234$ which was significantly higher $(P<0.001)$ than that of the normal control gps (PBS $18.50 \pm 2.023 \%$ and adjuvant gp $20.51 \pm 1.543 \%$ ).

4.10. Bm-iPGM Specific Cellular Adherence and Cytotoxicity to $M f$ and L3. Profound in vitro complement mediated cellular adherence and cytotoxicity to both Mf and L3 was noticed (Supplementary Figures 1(a) and 1(b)) in the presence of BmiPGM specific antibody. Percentage cytotoxicity was calculated by counting the number of immobile or dead parasites by adherence of effector cells against the total number of live parasites recovered which resulted in $61.4 \%$ and $52.0 \%$ death of Mf and L3, respectively, (Figure 10(a)) which was statistically highly significant $(P<0.001)$ when compared to normal Mastomys serum where no cell adhesion was seen. Interaction of anti-Bm-iPGM antibodies with $B$. malayi Mf (Figure 10(b)) and L3 (Figure 10(c)) was confirmed by fluorescence microscopy. 


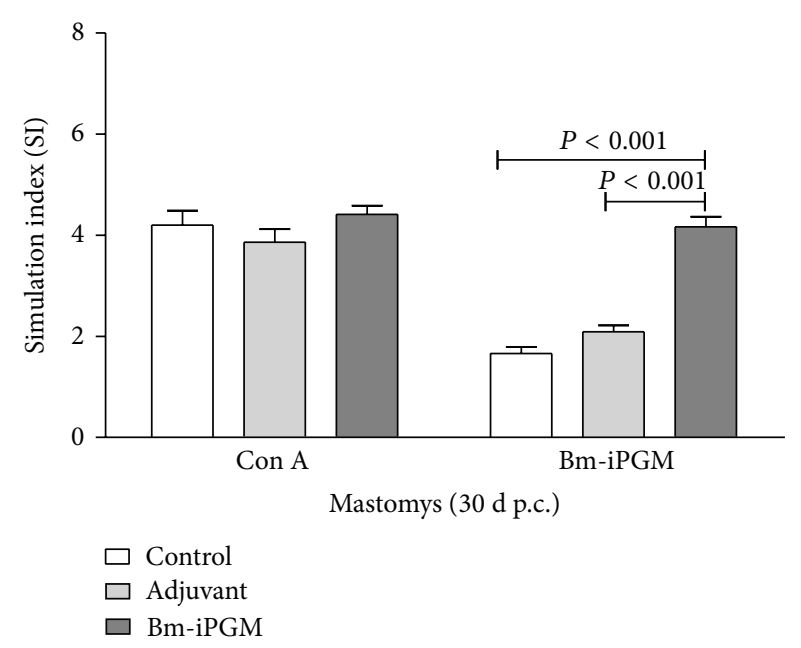

(a)

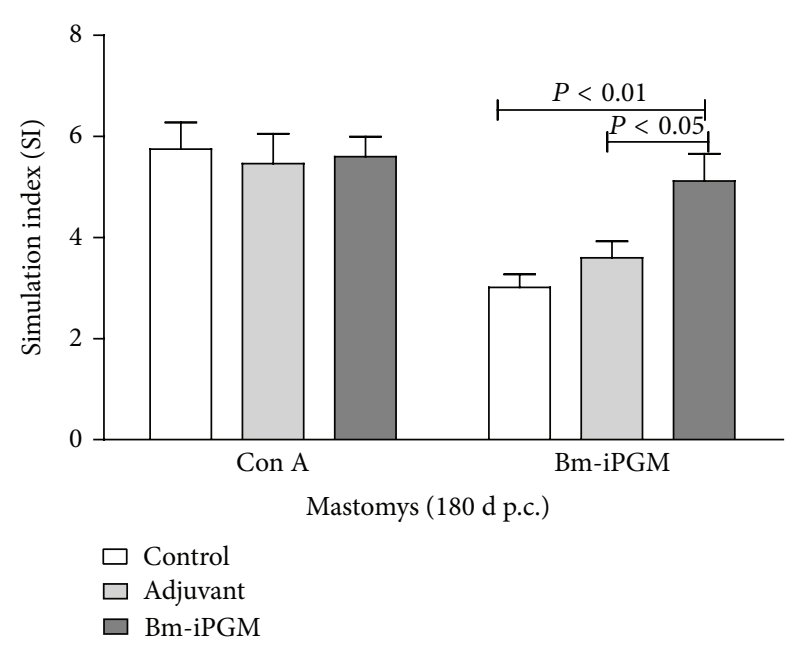

(b)

Figure 7: In vitro lymphocyte proliferation. Proliferation was assessed in the splenocytes isolated from immunized, adjuvant, and control group of Mastomys by radioactive incorporation of [3H]-thymidine after stimulation with either concanavalin A $(2.5 \mu \mathrm{g} / \mathrm{mL}) \mathrm{or}$ Bm-iPGM $(2.5 \mu \mathrm{g} / \mathrm{mL})$. Radioactive incorporation in the cells was measured and results are expressed as stimulation index. (a) Batch A: euthanized on day 30 after larval challenge. (b) Batch B: euthanized on day 180 after larval challenge.

\section{Discussion}

Phosphoglycerate mutases are the enzyme that catalyzes the reversible interconversion of 3-phosphoglycerate and 2-phosphoglycerate in both glycolysis and gluconeogenesis (Ulrike and Peter, 2007). B. malayi possesses cofactorindependent form of PGM while the dependent form is present in mammals that present iPGM as an attractive antifilarial drug or vaccine candidate. Raverdy [17] carried out the biochemical characterization of B. malayi iPGM and emphasized its worth as an antifilarial drug target. No information is, however, available on whether this protein plays any role in parasite immunobiology or host-parasite interactions.

In the present investigation, molecular and immune characterization studies of B. malayi iPGM have been carried out. Bm-iPGM was cloned, expressed, and purified to homogeneity as a single band protein of $\sim 60 \mathrm{kDa}$. Recombinant protein was found to be biochemically active in its native form as observed by circular dichroism and fluorescence spectroscopy which demonstrated $\alpha / \beta$ type topology having more than $50 \% \alpha$-helix and around $5-6 \% \beta$ strands which was consistent with the previous report on the three-dimensional crystal structure of iPGM of B. anthracis [26]. The homology model predicted the recombinant enzyme to be composed of a globular structure with two domains termed as the transferase and phosphatase interconnected by two short linker peptides. Both the domains demonstrate similar folds containing central $\beta$ sheet structure which are flanked on both sides by $\alpha$-helices, thus further confirming $\alpha$ - $\beta$ type topology. Till date, there are no known inhibitors of iPGM and the homology model generated can thus be utilised to design a series of inhibitors providing us with possible antifilarial drugs.
A small fragment of antigen can induce immune response against the whole antigen, thus locating promiscuous binding regions from the whole protein sequence can be useful in designing vaccine candidates. Bm-iPGM gene sequence in silico showed presence of 21 antigenic determinants carrying an average antigenic propensity of 1.0284 which points towards the high antigenicity of this protein. Further computational analysis of the target gene sequence predicted binding properties of the peptides to be driven by both MHC I and MHC II immune pathways. The alleles with high frequency within human population and with significant binding data were selected and the best binding peptides for each allele were identified and highlighted on the cartoon structure of BmiPGM and these were mainly located in the $\alpha$-helix region of the structure.

$\mathrm{Bm}$-iPGM seems indispensable for the parasite as it was found to be expressed by Mf, L3, and adult parasites and is also present in the excretory-secretory (ES) product of adult parasites. ES products released by live parasites can interfere with every aspect of host immunity [27] and requires functional characterization of their role in parasite and hostparasite interactions. Many of these proteins could serve as drug targets and can also be evaluated for prophylactic efficacy [11]; Bm-iPGM is one such protein and could serve to be major protein targeting all the important parasitic stages.

The serum collected from human bancroftian subjects and endemic normal individuals contained anti-Bm-iPGM antibodies which was demonstrated by reactivity against recombinant Bm-iPGM in blots. In ELISA, amicrofilaraemic symptomatic sera showed higher antibody titre to BmiPGM than endemic normals or microfilaraemic carriers while none of the 10 individual sera collected from filarial nonendemic area reacted with Bm-iPGM demonstrating filarial specificity of the recombinant protein which may also 

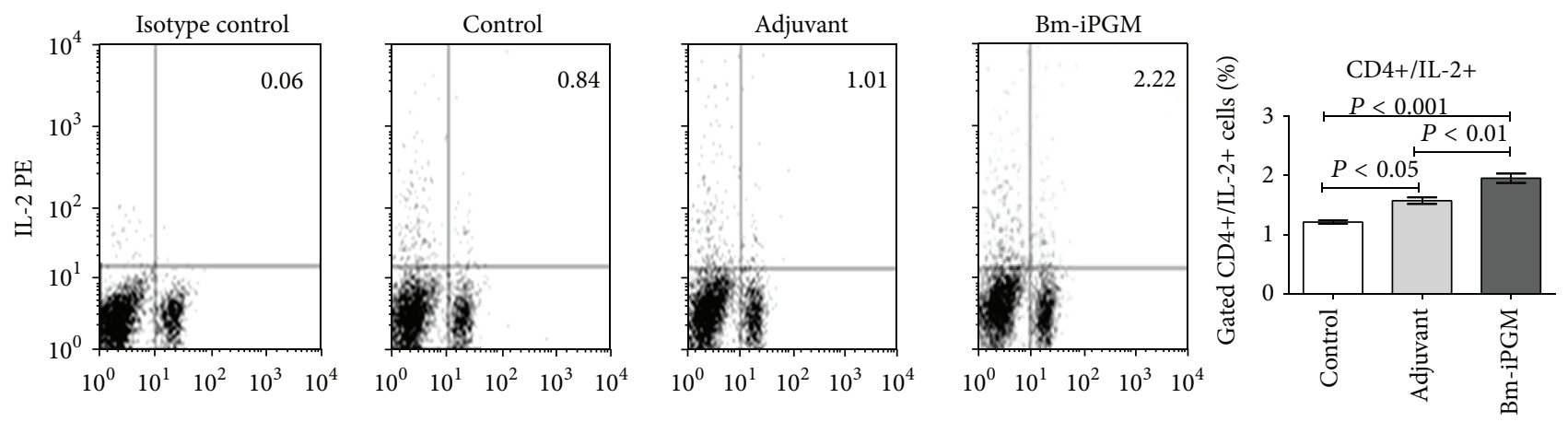

(a)
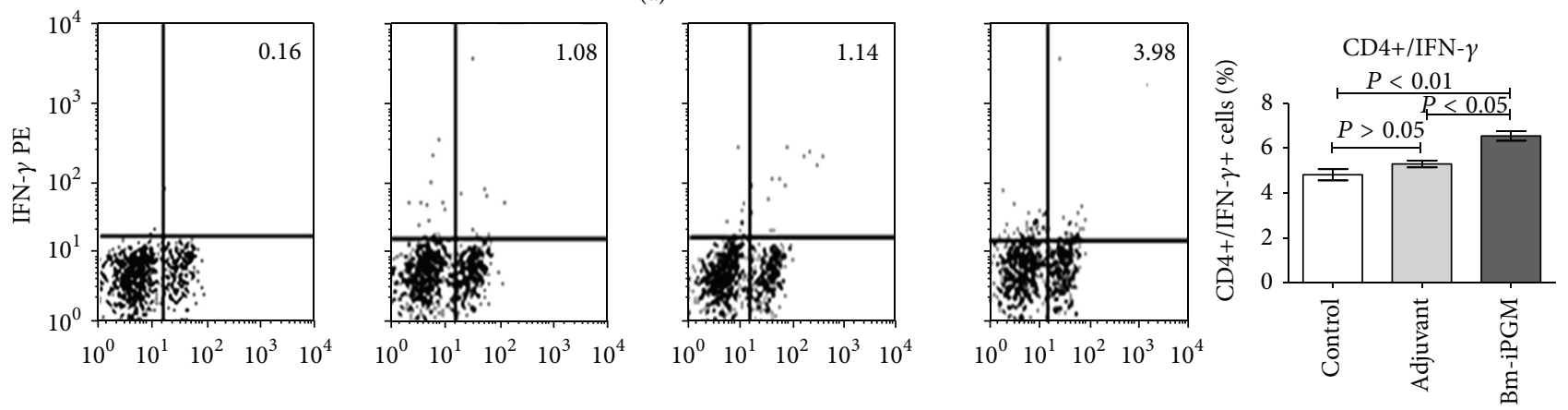

(b)
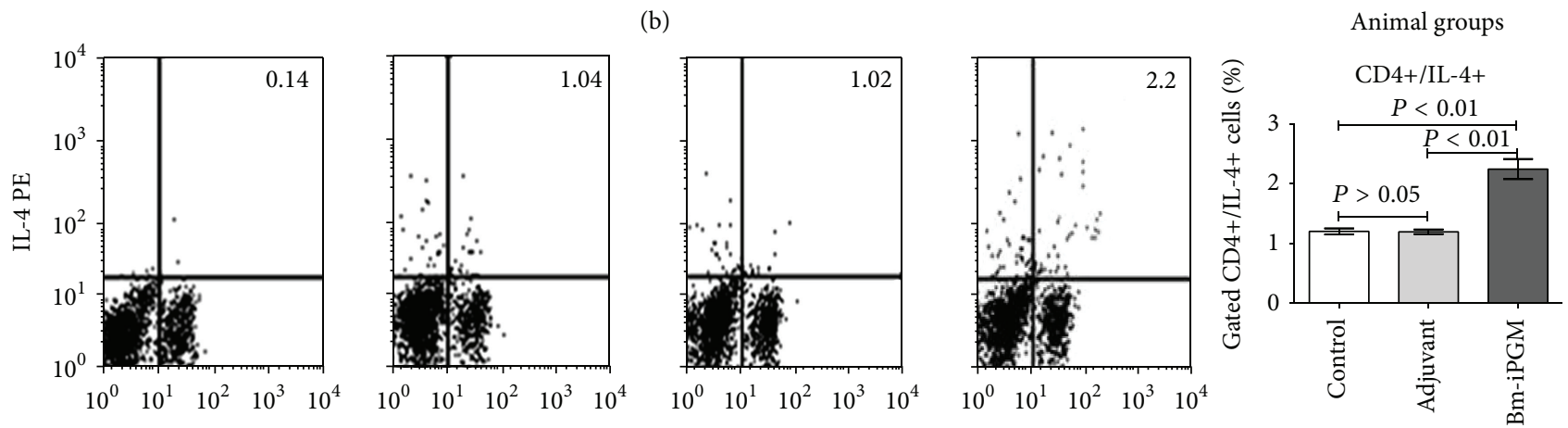

(c)
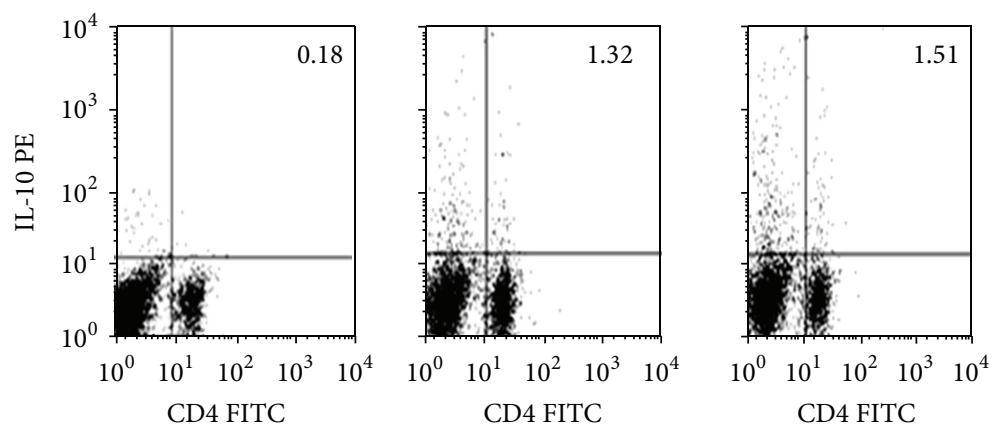

(d)

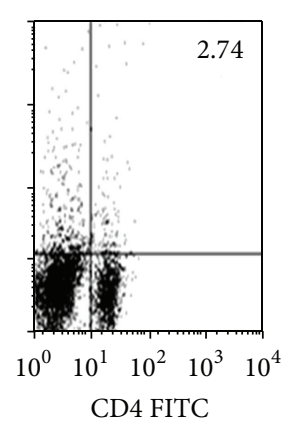

CD4 FITC

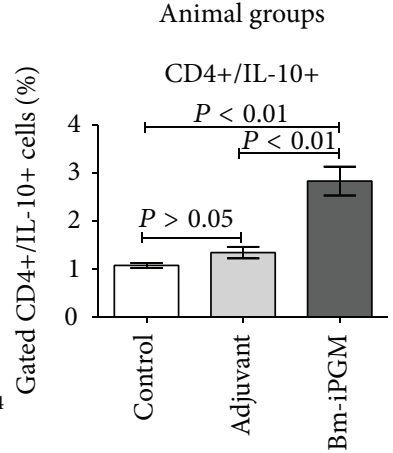

Animal groups

FIGURE 8: Flow cytometric analysis of intracellular IL-2, IFN- $\gamma$, IL-10, and IL-4 production in CD4+ T cells. Splenocytes were stained and processed as described in Section 2. Numbers in the upper right quadrant of dot plot represent the mean percentage of CD4+ T cells positive for (a) IL-2, (b) IFN- $\gamma$, (c) IL-4, and (d) IL-10 in particular group. Bar graph was generated for percentage of CD4+ T cells positive for IL-2, IFN- $\gamma$, IL-4, and IL-10. Statistical significance of the differences between mean values of immunized and control groups is depicted as ${ }^{*} P<0.05 ;{ }^{* *} P<0.01$; and ${ }^{* * *} P<0.001$. 


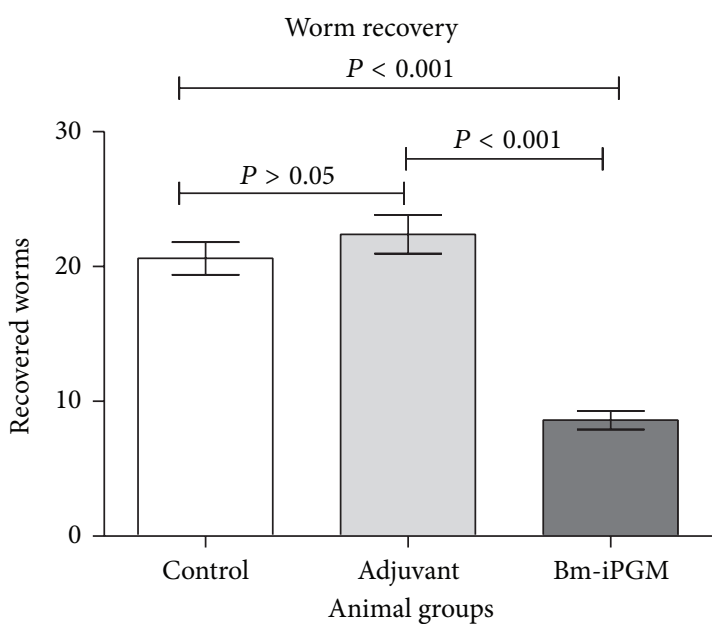

a)

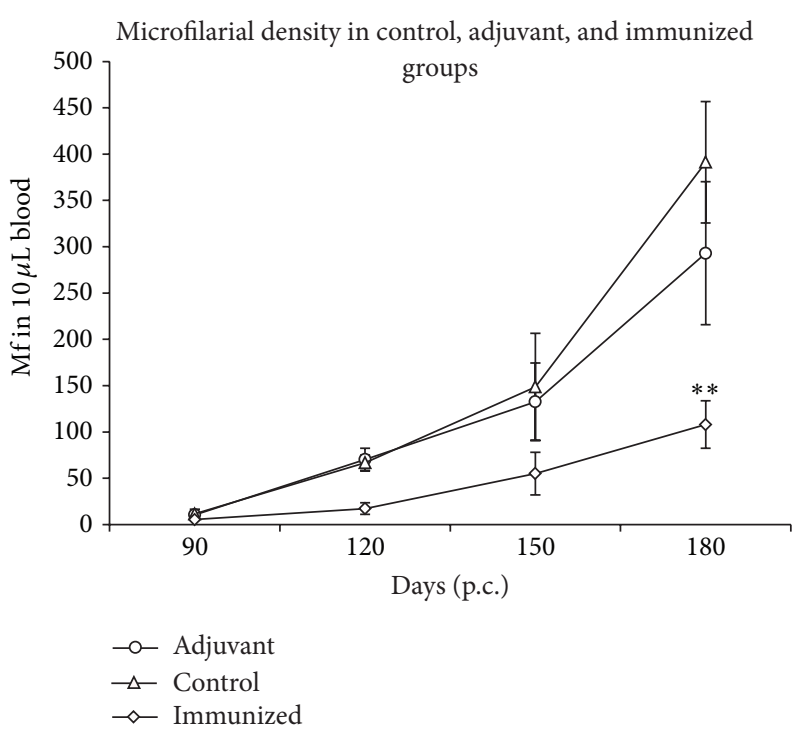

(b)

Figure 9: (a) Recovery of B. malayi preadults from different groups of Bm-iPGM immunized and control mice. Parasites were collected by washing the peritoneal cavity of infected BALB/c mice. Immunization of mice with Bm-iPGM resulted in marked reduction in worm establishment $(P<0.001)$. Statistical analysis was done using one-way ANOVA followed by Newman-Keuls Multiple Comparison Test. Each bar represents mean \pm S.E. of worms recovered from five animals. (b) Assessment of microfilarial density in tail blood of Bm-iPGM immunized adjuvant and control groups. Mf count was initiated from day 90 till day 180 p.c. Control and adjuvant groups exhibited elevated levels of blood Mf density. However, Mf levels remained low in Bm-iPGM immunized group and significant reduction in microfilarial density $(P<0.01 / 72.396 \%)$ on day 180 (p.c.) was observed in immunized animals. Each point represents a value obtained from six animals.

find its use in LF diagnosis. Seroreactivity with EN sera is of prime importance because EN are considered to be putatively immunoprotective and despite being continuously exposed to filarial larvae remain infection-free and do not develop the disease [28]. Few such antigens reacting strongly with EN sera have earlier been shown to offer protective immunity [2932]. We further investigated the nature of immune response generated after administration of recombinant Bm-iPGM in $\mathrm{BALB} / \mathrm{c}$ mice followed by prophylactic evaluation both in $\mathrm{BALB} / \mathrm{c}$ and Mastomys. It is worth mentioning that $\mathrm{BALB} / \mathrm{c}$ is immunologically a well dissected model and therefore has been used in the current investigation to decipher the type of immune responses triggered by the recombinant protein while Mastomys is a susceptible model that supports establishment of adult parasites mimicking the life cycle of parasite in human host. L3 are the most important stages in the life cycle of filariid that initiate an infection and further establish as adult parasites. Immunized mice were therefore challenged with L3 whose further development into L4 stage or adulthood was observed. Infection of BALB/c with B. malayi or B. pahangi L3 has earlier provided important insights into host-parasite biology in spite of the nonpermissiveness of immunocompetent mice to Brugia species [33].

An active filarial infection is characterised by downregulated Thl immune response in the form of suppressed $\mathrm{T}$ cell proliferation, decreased production of proinflammatory cytokines such as IFN- $\gamma$ and IL-2 along with Th2 dominated profile indicated by increased production of the $\operatorname{IgE}$ and Th2 cytokines IL-4, IL-5, IL-10, and IL-13 with expansion and greater mobilization of effector cells such as mast cells, eosinophils, and basophils [27,34-38]. Treg cells are induced by the parasite to evade the human immune system and are considered to be the important regulators of the immune response to filarial nematodes in experimental animals [39, 40]. CD4+ T cells express increased levels of CD25, CTLA4 , and glucocorticoid-induced TNF receptor family-related gene (GITR) with increased Treg functionality in microfilaraemic individuals. Treatment with antibodies to CD25 and GITR reverses this hyporesponsiveness with reduced parasite establishment [41] while depletion of Treg cells has shown to restore $\mathrm{T}$ cell as well as B cell proliferation [42].

Immunization with recombinant Bm-iPGM activated both the cellular and humoral arms of immunity. Profound antibody response was observed in $\mathrm{BALB} / \mathrm{c}$ with high IgG titers in addition to IgG1, IgG2a, IgG2b, IgG3, IgM, and IgA demonstrating induction of both Th1 and Th2 immune response which was supported by the equal ratio of IgG1 and IgG2a. Antifilarial antibodies have been reported to play an important role in protective immunity evidenced by studies where passive transfer of immune sera from resistant to naive animals showed reduced adult worm establishment [43]. B cell-deficient mice that lacked antibody displayed suppressed vaccine-induced protection against murine filariasis [44]. Antibodies directed against the surface of L3 and Mf have also been shown to be protective and an inverse correlation between adult worms and circulating antibodies has been noticed. IgG is believed to be the predominant antibody involved in antibody dependent cellular cytotoxicity 

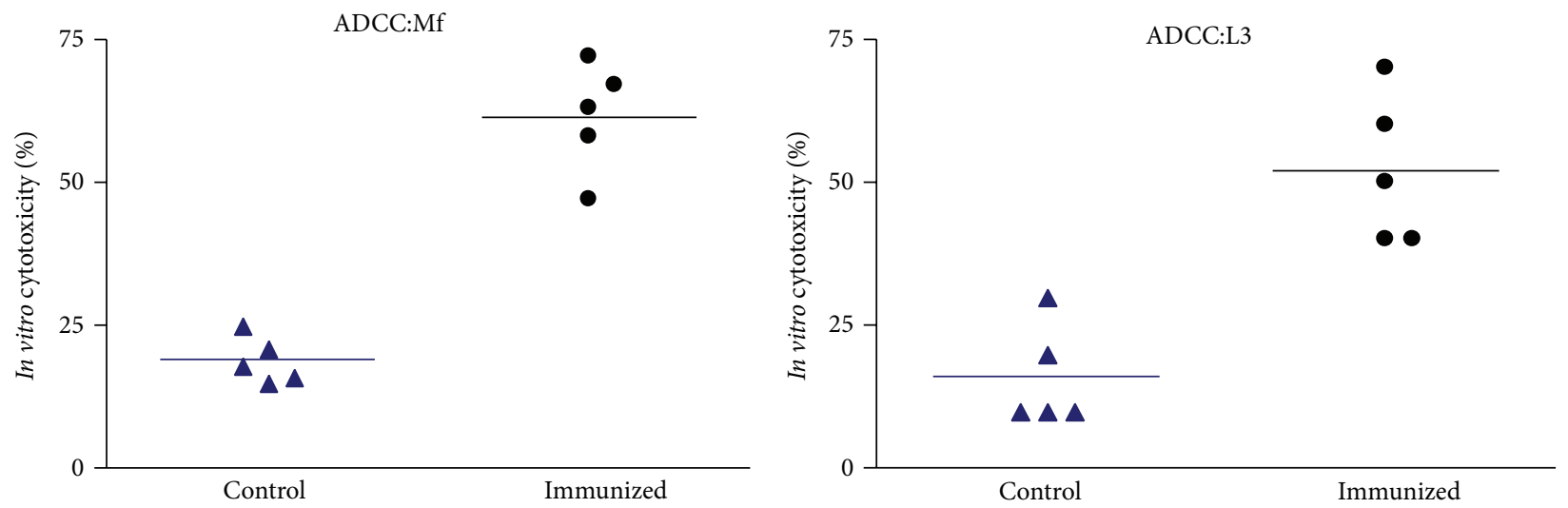

(a)

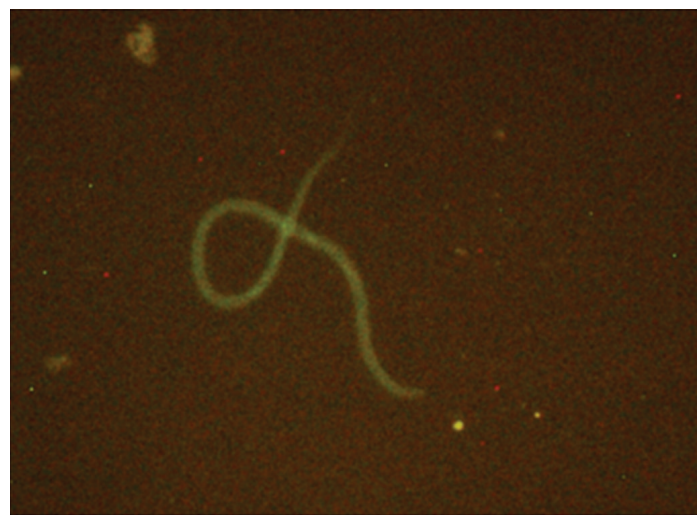

(b)

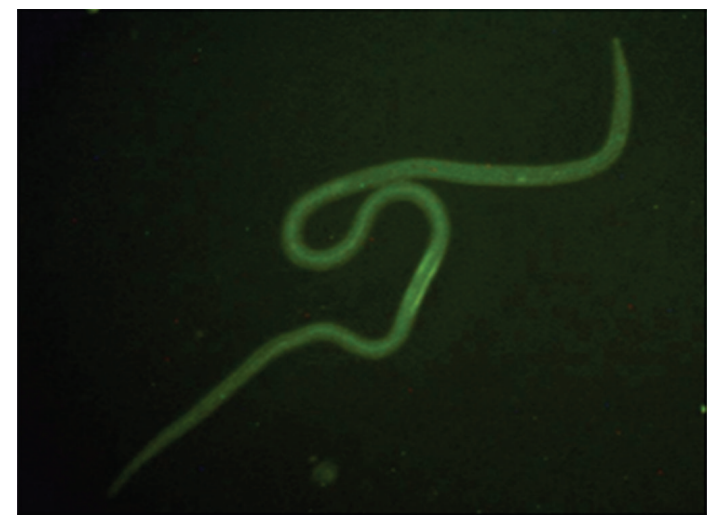

(c)

Figure 10: Antibody dependent cellular adhesion to Mf and L3 of B. malayi. Ten L3 and $100 \mathrm{Mf}$ were taken per well and were incubated with PEC isolated from normal Mastomys in the presence of sera from Bm-iPGM immunized animals. (a) Sera of Bm-iPGM immunized mice promoted adherence of PEC to Mf and L3 larvae and induced significant death of Mf (61.40\% cytotoxicity) and L3s (52\%). Photographs were captured on phase contrast microscope (Nikon, Japan) at 40x magnification. Data are presented as mean \pm S.E. values from five different wells. Interaction of anti-Bm-iPGM antibodies with B. malayi Mf (b) and L3 (c) as shown by fluorescence microscopy. Parasites were incubated with anti-Bm-iPGM sera for $4 \mathrm{~h}$ and further incubated with FITC labelled anti-mouse IgG for $2 \mathrm{~h}$. Images were captured under fluorescent microscope at 20X for Mf and 10X for L3.

(ADCC) mechanism involving adherence of neutrophils, macrophages, and eosinophils to Mf and L3 [45-48]. In our ADCC experiment peritoneal exudates cells got adhered on the surface of both L3 and Mf in presence of immunized sera causing parasite immobility and death. In a study on L. sigmodontis model of filariasis, it has been proposed that the cell recruitment depends on many factors like host susceptibility, immune response, and cell adhesion properties [49]. Immunofluorescence staining of L3 and Mf carried out with serum from immunized Mastomys also confirmed attachment of anti-Bm-iPGM antibodies to their surfaces confirming presence of iPGM on parasite surface and its interaction with antibodies. The reduction in parasite recovery and sterilization of recovered female worms from BmiPGM immunized animals could be due to this observed cytotoxicity to inoculated L3 which was apparent in BALB/c or both of the groups of Mastomys whether euthanized on day 30 after larval challenge or on day 180 p.c.

We observed significant levels of $\operatorname{IgA}$ which remains undefined in human bancroftian filariasis, in spite of the fact that studies in other helminths have indicated a protective role for parasite specific IgA restricting infection intensity [50-53]. Recently in human bancroftian filariasis, the role of $\operatorname{IgA}$ in protective immunity has been demonstrated [54]. IgA levels in mice have directly been found to be associated with raised interferon gamma (IFN- $\gamma$ ) production by $\mathrm{T}$ cells $[10,54]$. The role of IgM, remains undefined in nematodes; however, in vitro, it has been shown to play a major role in adherence of host immune cells to filarial L3 and Mf causing cytotoxicity and their death [55]. Thus, elevated level of Bm-iPGM specific IgM might also have adversely affected the survival of challenged B. malayi L3 and their further development in Mastomys.

Raised reactive oxygen species (ROS) levels might have been one of the mechanisms responsible for providing protection $[56,57]$ that could be correlated with the IFN- $\gamma$ levels in the immunized mice since macrophage activation largely depends upon IFN- $\gamma$ produced by Th1 (CD8+T cells). Animals from both the batches of Bm-iPGM immunized Mastomys revealed higher $\mathrm{T}$ cell proliferation in presence 
of recombinant Bm-iPGM or mitogen Con A conferring that Bm-iPGM was equally effective in causing cellular proliferation as Con A. Mice deficient in T and B cells have been shown to be permissive to filarial infection, thereby stressing the importance of $\mathrm{T}$ and $\mathrm{B}$ cells in preventing filarial establishment [58-60]. An expansion in B cell population was also noticed after Bm-iPGM administration in the animals as observed by the increased levels of CD19+ B cells. Low microfilaraemia in antigen immunized group which was apparent from day 120 onwards may also be an attribute for $\mathrm{T}$ cell proliferation as state of hyporesponsiveness has been directly linked to high number of circulating Mfs [61]. Significant upregulation in CD4+ and CD8+ cells population was noticed in Bm-iPGM immunized animals, which are in general used for defining helper and cytotoxic $\mathrm{T}$ cell subpopulations, respectively, $[62,63]$. It is generally believed that a vaccine will have at its core instigation of an antigen specific CD4+ T cell response which plays an important role in development of protective immunity against infection. A number of studies have shown that CD $4+\mathrm{T}$ cells play a critical role in regulating the immune response to nematode parasites where depletion of CD4+ T cells in infected mice has been shown to enhance adult worm and microfilarial burden.

Contradictions to the studies that consider immune responses to helminth parasites including filariids to be Th2 type do exist and reports either impairment of both Th1 and Th2 pathways and domination of Th1 response [64, 65]; these differing observations might have been due to different life stages examined. Past studies in murine models advocate the involvement of both Th1 and Th2 arms of immunity in resistance to filarial parasites [66, 67]; thus, downregulation in Th1/Th2 effector function would certainly facilitate the establishment and maintenance of filarial infections. Therefore, any vaccine regimen that would help to overcome downregulation might be useful in impairing the establishment of filarial parasites.

Immunization with Bm-iPGM led to generation of an effective immune mechanism mediated through an upregulated Th1 (IFN- $\gamma$, IL-2) and Th2 (IL-4, IL-10) cytokine production that could provide considerable protection $(58 \%$ : $\mathrm{BALB} / \mathrm{c}$; 65-68\%: Mastomys) against challenged larval development possibly by combating the immune downregulation caused by the challenged larvae. Since BALB/c were euthanized on day 15 after L3 challenge, all the L3 stages had converted into advanced L4 stages and none of the recovered larva was L3. However, this period could demonstrate well that there was noticeable killing of the L3 in Bm-iPGM immunized mice as was observed in the Mastomys which displayed reduction in parasite establishment both on day 30 or day 180 p.c.

IL- 4 and IL- 5 have been shown to play critical roles in the host resistance to $L$. loa infection in knock out BALB/C mice (Nicholas 2012); Thl cytokine IFN- $\gamma$ controls B. malayi infection in murine models; IL-5 controls adult worm development in primary infection and IL- 4 mediated pathways are necessary for the control of $\mathrm{Mf}$ and the development of adult worms [68]. Studies in IL-4 knockout mice have revealed an undeniable role of IL-4 in countering larval establishment in murine modelof Litomosoides sigmodontis, diminished Th2-type responses with failure to produce parasite specific IgGlin Trichuris muris infection [69-71]. IL4 dependent effector mechanisms have been shown to be dependent on IL-10 in mice that were knocked out for IL4/IL-10 displaying antagonistic activity between IL-4 and IL10 [68]. Recently, levels of IL-10 have been directly linked to parasite survival, overcome resistance, and allow full patency in murine filariasis [72]. Immunity in human infections has been reported to be associated with an elevated level of IL-2 and IFN- $\gamma[73,74]$. EN and chronic patients develop stronger immune response, raised IFN- $\gamma$ level as compared to that of patients carrying active filarial infection $[75,76]$. B. malayi Mf, and L3 have been shown to be killed in vitro by IFN- $\gamma$ activated macrophages via production of nitric oxide (NO) and ROS [77-79]. A mixed Th1/Th2 response as observed in the current investigation has been ascribed to exert profound immune protective function $[80,81]$. The present findings clearly suggests that, on vaccination with Bm-iPGM, a correct milieu with a mixed type of Th1/Th2 immune response accompanied with innate immunity was maintained which was efficient in providing significant degree of protection against establishment of B. malayi in the immunized host. The different molecular events that are required to maintain a balanced cytokine levels need careful investigation to further facilitate vaccine development programme.

In summary, the overall immune response generated by Bm-iPGM correlated with the percentage level of protection achieved in terms of low adult worm recovery, reduced microfilaraemia, and embryostatic effect in female worms. The independent phosphoglycerate mutase of filarial parasite B. malayi appears to be an immunogenic protein with diagnostic potential which considerably impairs filarial parasite establishment and presents a promising vaccine candidate. Immunization studies with Bm-iPGM using human administrable adjuvants are underway to further improve its efficacy and usefulness.

\section{Conflict of Interests}

The authors declare that they have no conflict of interests.

\section{Acknowledgments}

The authors are grateful to Mr. A. L. Vishwakarma (SAIF division, CSIR-CDRI,) for his help in acquiring flow cytometry data and to Mr. A. K. Roy and Mr. R. N. Lal for experimental maintenance of $B$. malayi. They are grateful to CSIR and UGC for providing financial assistance to Prashant K. Singh, Susheela Kushwaha, and Ajay K. Rana in the form of research fellowships. The authors also acknowledge CSIR for financial assistance in the form of CSIR Network Project SPLenDID (BSC 0104). This papers bears CSIR-CDRI communication no. 8701 .

\section{References}

[1] E. Palumbo, "Filariasis: diagnosis, treatment and prevention," Acta Biomedica de l'Ateneo Parmense, vol. 79, no. 2, pp. 106-109, 2008. 
[2] Progress Report 2000-2009 and Strategic Plan 2010-2020 of the Global Programme to Eliminate Lymphatic filariasis: Halfway Towards Eliminating Lymphatic filariasis, 2010.

[3] S. Sabesan, M. Palaniyandi, P. K. Das, and E. Michael, "Mapping of lymphatic filariasis in India," Annals of Tropical Medicine and Parasitology, vol. 94, no. 6, pp. 591-606, 2000.

[4] D. H. Molyneux, "Filaria control and elimination: diagnostic, monitoring and surveillance needs," Transactions of the Royal Society of Tropical Medicine and Hygiene, vol. 103, no. 4, pp. 338341, 2009.

[5] WHO, "Global programme to eliminate lymphatic filariasis. progress report on mass drug administration in 2008," in Weekly Epidemiological Record, vol. 42, pp. 437-444, World Health Organization, Geneva, Switzerland, 2009.

[6] E. Ghedin, S. Wang, D. Spiro et al., "Draft genome of the filarial nematode parasite Brugia malayi," Science, vol. 317, no. 5845, pp. 1756-1760, 2007.

[7] J. van der Oost, M. A. Huynen, and C. H. Verhees, "Molecular characterization of phosphoglycerate mutase in archaea," FEMS Microbiology Letters, vol. 212, no. 1, pp. 111-120, 2002.

[8] U. Johnsen and P. Schönheit, "Characterization of cofactordependent and cofactor-independent phosphoglycerate mutases from Archaea," Extremophiles, vol. 11, no. 5, pp. 647-657, 2007.

[9] M. W. Nowicki, B. Kuaprasert, I. W. McNae et al., "Crystal structures of Leishmania mexicana phosphoglycerate mutase suggest a one-metal mechanism and a new enzyme subclass," Journal of Molecular Biology, vol. 394, no. 3, pp. 535-543, 2009.

[10] Y. Zhang, S. Pacheco, C. L. Acuna et al., "Immunoglobulin Adeficient mice exhibit altered $\mathrm{T}$ helper 1-type immune responses but retain mucosal immunity to influenza virus," Immunology, vol. 105, no. 3, pp. 286-294, 2002.

[11] S. Bennuru, R. Semnani, Z. Meng, J. M. C. Ribeiro, T. D. Veenstra, and T. B. Nutman, "Brugia malayi excreted/secreted proteins at the host/parasite interface: stage- and genderspecific proteomic profiling," PLoS Neglected Tropical Diseases, vol. 3, no. 4, article e410, 2009.

[12] U. Singh, S. Misra, P. K. Murthy, J. C. Katiyar, A. Agrawal, and A. R. Sircar, "Immunoreactive molecules of Brugia malayi and their diagnostic potential," Serodiagnosis and Immunotherapy in Infectious Disease, vol. 8, no. 3-4, pp. 207-212, 1997.

[13] L. A. Kelley and M. J. E. Sternberg, "Protein structure prediction on the web: a case study using the Phyre server," Nature Protocols, vol. 4, no. 3, pp. 363-371, 2009.

[14] A. S. Devi, T. Rehana, A. S. Kolaskar, and M. W. Pandit, "Hydrophilicity and antigenicity of proteins-a case study of myoglobin and hemoglobin," Journal of Biosciences, vol. 14, no. 2, pp. 133-142, 1989.

[15] H. Singh and G. P. S. Raghava, "ProPred: prediction of HLA-DR binding sites," Bioinformatics, vol. 17, no. 12, pp. 1236-1237, 2002.

[16] H. Singh and G. P. S. Raghava, "ProPred1: prediction of promiscuous MHC class-I binding sites," Bioinformatics, vol. 19, no. 8, pp. 1009-1014, 2003.

[17] S. Raverdy, Y. Zhang, J. Foster, and C. K. S. Carlow, "Molecular and biochemical characterization of nematode cofactor independent phosphoglycerate mutases," Molecular and Biochemical Parasitology, vol. 156, no. 2, pp. 210-216, 2007.

[18] M. M. Bradford, "A rapid and sensitive method for the quantitation of microgram quantities of protein utilizing the principle of protein dye binding," Analytical Biochemistry, vol. 72, no. 1-2, pp. 248-254, 1976.
[19] H. Towbin, T. Staehelin, and J. Gordon, "Electrophoretic transfer of proteins from polyacrylamide gels to nitrocellulose sheets: procedure and some applications," Proceedings of the National Academy of Sciences of the United States of America, vol. 76, no. 9, pp. 4350-4354, 1979.

[20] S. Vedi, A. Dangi, K. Hajela, and S. Misra-Bhattacharya, "Vaccination with $73 \mathrm{kDa}$ recombinant heavy chain myosin generates high level of protection against Brugia malayi challenge in jird and mastomys models," Vaccine, vol. 26, no. 47, pp. 5997-6005, 2008.

[21] M. Singh, S. Shakya, V. K. Soni, A. Dangi, N. Kumar, and S.-M. Bhattacharya, "The n-hexane and chloroform fractions of Piper betle L. trigger different arms of immune responses in BALB/c mice and exhibit antifilarial activity against human lymphatic filarid Brugia malayi," International Immunopharmacology, vol. 9, no. 6, pp. 716-728, 2009.

[22] N. Zurgil, Y. Shafran, E. Afrimzon, D. Fixler, A. Shainberg, and M. Deutsch, "Concomitant real-time monitoring of intracellular reactive oxygen species and mitochondrial membrane potential in individual living promonocytic cells," Journal of Immunological Methods, vol. 316, no. 1-2, pp. 27-41, 2006.

[23] S. Misra, M. Mukherjee, M. Dikshit, and R. K. Chatterjee, "Cellular immune response of mastomys and gerbils in experimental filariasis," Tropical Medicine and International Health, vol. 3, no. 2, pp. 124-129, 1998.

[24] A. Ayuso-Sacido, C. Graham, J. P. Greenfield, and J. A. Boockvar, "The duality of epidermal growth factor receptor (EGFR) signaling and neural stem cell phenotype: cell enhancer or cell transformer?" Current Stem Cell Research \& Therapy, vol. 1, no. 3, pp. 387-394, 2006.

[25] J. T. Yang, C.-S. C. Wu, and H. M. Martinez, "Calculation of protein conformation from circular dichroism," Methods in Enzymology, vol. 130, pp. 208-269, 1986.

[26] M. Nukui, L. V. Mello, J. E. Littlejohn et al., "Structure and molecular mechanism of Bacillus anthracis cofactorindependent phosphoglycerate mutase: a crucial enzyme for spores and growing cells of Bacillus species," Biophysical Journal, vol. 92, no. 3, pp. 977-988, 2007.

[27] J. P. Hewitson, J. R. Grainger, and R. M. Maizels, "Helminth immunoregulation: the role of parasite secreted proteins in modulating host immunity," Molecular and Biochemical Parasitology, vol. 167, no. 1, pp. 1-11, 2009.

[28] E. A. Ottesen, "The Wellcome Trust Lecture. Infection and disease in lymphatic filariasis: an immunological perspective," Parasitology, vol. 104, pp. S71-S79, 1992.

[29] D. O. Freedman, T. B. Nutman, and E. A. Ottesen, "Protective immunity in bancroftian filariasis. Selective recognition of a 43-kD larval stage antigen by infection-free individuals in an endemic area," Journal of Clinical Investigation, vol. 83, no. 1, pp. 14-22, 1989.

[30] S. Lustigman, E. R. James, W. Tawe, and D. Abraham, "Towards a recombinant antigen vaccine against Onchocerca volvulus," Trends in Parasitology, vol. 18, no. 3, pp. 135-141, 2002.

[31] A. J. Macdonald, W. Tawe, O. Leon et al., "Ov-ASP-1, the Onchocerca volvulus homologue of the activation associated secreted protein family is immunostimulatory and can induce protective anti-larval immunity," Parasite Immunology, vol. 26, no. 1, pp. 53-62, 2004.

[32] S. Ramachandran, M. P. Kumar, R. M. V. Rami et al., "The larval specific lymphatic filarial ALT-2: induction of protection using protein or DNA vaccination," Microbiology and Immunology, vol. 48, no. 12, pp. 945-955, 2004. 
[33] A. Hörauf and B. Fleischer, "Immune responses to filarial infection in laboratory mice," Medical Microbiology and Immunology, vol. 185, no. 4, pp. 207-215, 1997.

[34] E. A. Ottesen, P. F. Weller, and L. Heck, "Specific cellular immune unresponsiveness in human filariasis," Immunology, vol. 33, no. 3, pp. 413-421, 1977.

[35] J. L. Grogan, P. G. Kremsner, A. M. Deelder, and M. Yazdanbakhsh, "Elevated proliferation and interleukin-4 release from $\mathrm{CD}^{+}$cells after chemotherapy in human Schistosoma haematobium infection," European Journal of Immunology, vol. 26, no. 6, pp. 1365-1370, 1996.

[36] R. M. Maizels, A. Balic, N. Gomez-Escobar, M. Nair, M. D. Taylor, and J. E. Allen, "Helminth parasites-masters of regulation," Immunological Reviews, vol. 201, pp. 89-116, 2004.

[37] J. M. Behnke, C. J. Barnard, and D. Wakelin, "Understanding chronic nematode infections: evolutionary considerations, current hypotheses and the way forward," International Journal for Parasitology, vol. 22, no. 7, pp. 861-907, 1992.

[38] R. M. Maizels and M. Yazdanbakhsh, "Immune regulation by helminth parasites: cellular and molecular mechanisms," Nature Reviews Immunology, vol. 3, no. 9, pp. 733-744, 2003.

[39] Y. Belkaid, "Regulatory $\mathrm{T}$ cells and infection: a dangerous necessity," Nature Reviews Immunology, vol. 7, no. 11, pp. 875888, 2007.

[40] M. D. Taylor, N. van der Werf, A. Harris et al., "Early recruitment of natural $\mathrm{CD}^{+}{ }^{+}$Foxp $^{+}$Treg cells by infective larvae determines the outcome of filarial infection," European Journal of Immunology, vol. 39, no. 1, pp. 192-206, 2009.

[41] M. D. Taylor, L. LeGoff, A. Harris, E. Malone, J. E. Allen, and R. M. Maizels, "Removal of regulatory $\mathrm{T}$ cell activity reverses hyporesponsiveness and leads to filarial parasite clearance in vivo," Journal of Immunology, vol. 174, no. 8, pp. 4924-4933, 2005.

[42] L. J. Wammes, F. Hamid, A. E. Wiria et al., "Regulatory T cells in human lymphatic filariasis: stronger functional activity in microfilaremics," PLoS Neglected Tropical Diseases, vol. 6, no. 5, Article ID e1655, 2012.

[43] V. Chenthamarakshan, K. Cheirmaraj, M. V. R. Reddy, and B. C. Harinath, "Immunoprophylactic studies with a $43 \mathrm{kDa}$ human circulating filarial antigen and a cross reactive $120 \mathrm{kDa}$ Brugia malayi sodium dodecyl sulphate soluble antigen in filariasis," Journal of Biosciences, vol. 22, no. 1, pp. 91-98, 1997.

[44] C. Martin, M. Saeftel, P. N. Vuong et al., "B-cell deficiency suppresses vaccine-induced protection against murine filariasis but does not increase the recovery rate for primary infection," Infection and Immunity, vol. 69, no. 11, pp. 7067-7073, 2001.

[45] N. Weiss and M. Tanner, "Studies on Dipetalonema viteae (Filarioidea). 3. Antibody-dependent cell-mediated destruction of microfiliariae in vivo," Tropenmedizin und Parasitologie, vol. 30, no. 1, pp. 73-80, 1979.

[46] R. Chandrashekar, U. R. Rao, P. B. Parab, and D. Subrahmanyam, "Brugia malayi: serum dependent cell-mediated reactions to microfilariae," Southeast Asian Journal of Tropical Medicine and Public Health, vol. 16, no. 1, pp. 15-21, 1985.

[47] R. Chandrashekar, U. R. Rao, and D. Subrahmanyam, "Serum dependent cell-mediated immune reactions to Brugia pahangi infective larvae," Parasite Immunology, vol. 7, no. 6, pp. 633-641, 1985.

[48] B. K. L. Sim, B. H. Kwa, and J. W. Mak, "Immune responses in human Brugia malayi infections: serum dependent cellmediated destruction of infective larvae in vitro," Transactions of the Royal Society of Tropical Medicine and Hygiene, vol. 76, no. 3, pp. 362-370, 1982.

[49] T. Attout, C. Martin, S. A. Babayan et al., "Pleural cellular reaction to the filarial infection Litomosoides sigmodontis is determined by the moulting process, the worm alteration, and the host strain," Parasitology International, vol. 57, no. 2, pp. 201211, 2008.

[50] D. C. Lloyd, R. J. Purrott, E. J. Reeder, A. A. Edwards, and G. W. Dolphin, "Chromosome aberrations induced in human lymphocytes by radiation from 252Cf," International Journal of Radiation Biology, vol. 34, no. 2, pp. 177-186, 1978.

[51] N. M. Almond and R. M. E. Parkhouse, "Immunoglobulin class specific responses to biochemically defined antigens of Trichinella spiralis," Parasite Immunology, vol. 8, no. 4, pp. 391406, 1986.

[52] J.-M. Grzych, D. Grezel, J.-L. Neyrinck et al., "IgA antibodies to a protective antigen in human Schistosomiasis mansoni," Journal of Immunology, vol. 150, no. 2, pp. 527-535, 1993.

[53] H. M. Khalil, M. H. Abd el Baki, M. M. Abd el Mawla, K. M. Maklad, S. A. Sharaf, and A. E. Saad, "Interleukin-4, immunoglobulin $\mathrm{E}$ and immunoglobulin $\mathrm{A}$ and resistance to re-infection with Schistosoma haematobium before and after chemotherapy," Journal of the Egyptian Society of Parasitology, vol. 29, no. 2, pp. 395-408, 1999.

[54] B. R. Sahu, M. C. Mohanty, P. K. Sahoo, A. K. Satapathy, and B. Ravindran, "Protective immunity in human filariasis: a role for parasite-specific IgA responses," Journal of Infectious Diseases, vol. 198, no. 3, pp. 434-443, 2008.

[55] B. Rajan, T. Ramalingam, and T. V. Rajan, "Critical role for IgM in host protection in experimental filarial infection," Journal of Immunology, vol. 175, no. 3, pp. 1827-1833, 2005.

[56] H. L. Callahan, R. K. Crouch, and E. R. James, "Helminth anti-oxidant enzymes: a protective mechanism against host oxidants?” Parasitology Today, vol. 4, no. 8, pp. 218-225, 1988.

[57] P. M. Brophy and D. I. Pritchard, "Immunity to helminths: ready to tip the biochemical balance?" Parasitology Today, vol. 8, no. 12, pp. 419-422, 1992.

[58] S. Babu, L. D. Shultz, T. R. Klei, and T. V. Rajan, "Immunity in experimental murine filariasis: roles of T and B cells revisited," Infection and Immunity, vol. 67, no. 6, pp. 3166-3167, 1999.

[59] A. L. Vincent, A. C. Vickery, A. Winters, and W. A. Sodeman Jr., "Life cycle of Brugia pahangi (Nematoda) in nude mice, $\mathrm{C} 3 \mathrm{H} / \mathrm{HeN}$ (nu/nu)," Journal of Parasitology, vol. 68, no. 4, pp. 553-560, 1982.

[60] R. R. Suswillo, D. G. Owen, and D. A. Denham, "Infections of Brugia pahangi in conventional and nude (athymic) mice," Acta Tropica, vol. 37, no. 4, pp. 327-335, 1980.

[61] R. A. O'Connor, J. S. Jenson, J. Osborne, and E. Devaney, "An enduring association? Microfilariae and immunosupression in lymphatic filariasis," Trends in Parasitology, vol. 19, no. 12, pp. 565-570, 2003.

[62] W. Ellmeier, S. Sawada, and D. R. Littman, "The regulation of CD4 and CD8 coreceptor gene expression during T cell development," Annual Review of Immunology, vol. 17, pp. 523554, 1999.

[63] A. Dhur, P. Galan, P. Preziosi, and S. Hercberg, "Lymphocyte subpopulations in the thymus, lymph nodes and spleen of irondeficient and rehabilitated mice," Journal of Nutrition, vol. 121, no. 9, pp. 1418-1424, 1991.

[64] S. Babu, C. P. Blauvelt, V. Kumaraswami, and T. B. Nutman, "Regulatory networks induced by live parasites impair both Th1 
and Th2 pathways in patent lymphatic filariasis: implications for parasite persistence," Journal of Immunology, vol. 176, no. 5, pp. 3248-3256, 2006.

[65] S. Babu and T. B. Nutman, "Proinflammatory cytokines dominate the early immune response to filarial parasites," Journal of Immunology, vol. 171, no. 12, pp. 6723-6732, 2003.

[66] S. Babu, L. M. Ganley, T. R. Klei, L. D. Shultz, and T. V. Rajan, "Role of gamma interferon and interleukin-4 in host defense against the human filarial parasite Brugia malayi," Infection and Immunity, vol. 68, no. 5, pp. 3034-3035, 2000.

[67] T. V. Rajan, P. Porte, J. A. Yates, L. Keeper, and L. D. Shultz, "Role of nitric oxide in host defense against an extracellular, metazoan parasite, Brugia malayi," Infection and Immunity, vol. 64, no. 8, pp. 3351-3353, 1996.

[68] S. Specht, L. Volkmann, T. Wynn, and A. Hoerauf, "Interleukin10 (IL-10) counterregulates IL-4-dependent effector mechanisms in murine filariasis," Infection and Immunity, vol. 72, no. 11, pp. 6287-6293, 2004.

[69] T. A. Wynn, A. Reynolds, S. James et al., "IL-12 enhances vaccine-induced immunity to schistosomes by augmenting both humoral and cell-mediated immune responses against the parasite," Journal of Immunology, vol. 157, no. 9, pp. 4068-4078, 1996.

[70] A. J. Bancroft, D. Artis, D. D. Donaldson, J. P. Sypek, and R. K. Grencis, "Gastrointestinal nematode expulsion in IL4 knockout mice is IL-13 dependent," European Journal of Immunology, vol. 30, no. 7, pp. 2083-2091, 2000.

[71] L. Le Goff, T. J. Lamb, A. L. Graham, Y. Harcus, and J. E. Allen, "IL-4 is required to prevent filarial nematode development in resistant but not susceptible strains of mice," International Journal for Parasitology, vol. 32, no. 10, pp. 1277-1284, 2002.

[72] S. Specht, M. D. Taylor, M. A. Hoeve, J. E. Allen, R. Lang, and A. Hoerauf, "Over expression of IL-10 by macrophages overcomes resistance to murine filariasis," Experimental Parasitology, vol. 132, no. 1, pp. 90-96, 2012.

[73] L. H. Elson, M. H. Calvopina, W. Y. Paredes et al., "Immunity to onchocerciasis: putative immune persons produce a Thl-like response to Onchocerca volvulus," Journal of Infectious Diseases, vol. 171, no. 3, pp. 652-658, 1995.

[74] K. A. Dimock, M. L. Eberhard, and P. J. Lammie, "Th1-like antifilarial immune responses predominate in antigen-negative persons," Infection and Immunity, vol. 64, no. 8, pp. 2962-2967, 1996.

[75] R. M. Maizels, E. Sartono, A. Kurniawan, F. Partono, M. E. Selkirk, and M. Yazdanbakhsh, "T-cell activation and the balance of antibody isotypes in human lymphatic filariasis," Parasitology Today, vol. 11, no. 2, pp. 50-56, 1995.

[76] E. Sartono, Y. C. M. Kruize, A. Kurniawan et al., "Elevated cellular immune responses and interferon- $\gamma$ release after longterm diethylcarbamazine treatment of patients with human lymphatic filariasis," Journal of Infectious Diseases, vol. 171, no. 6, pp. 1683-1687, 1995.

[77] M. J. Taylor, H. F. Cross, A. A. Mohammed, A. J. Trees, and A. E. Bianco, "Susceptibility of Brugia malayi and Onchocerca lienalis microfilariae to nitric oxide and hydrogen peroxide in cell-free culture and from IFN $\gamma$-activated macrophages," Parasitology, vol. 112, part 3, pp. 315-322, 1996.

[78] G. R. Thomas, M. McCrossan, and M. E. Selkirk, "Cytostatic and cytotoxic effects of activated macrophages and nitric oxide donors on Brugia malayi," Infection and Immunity, vol. 65, no. 7, pp. 2732-2739, 1997.
[79] A. W. Pfaff, H. Schulz-Key, P. T. Soboslay, S. M. Geiger, and W. $\mathrm{H}$. Hoffmann, "The role of nitric oxide in the innate resistance to microfilariae of Litomosoides sigmodontis in mice," Parasite Immunology, vol. 22, no. 8, pp. 397-405, 2000.

[80] A. El Bouhdidi, C. Truyens, M.-T. Rivera, H. Bazin, and Y. Carlier, "Trypanosoma cruzi infection in mice induces a polyisotypic hypergammaglobulinaemia and parasite-specific response involving high IgG2a concentrations and highly avid IgG1 antibodies," Parasite Immunology, vol. 16, no. 2, pp. 69-76, 1994.

[81] B. Pulendran, J. L. Smith, G. Caspary et al., "Distinct dendritic cell subsets differentially regulate the class of immune response in vivo," Proceedings of the National Academy of Sciences of the United States of America, vol. 96, no. 3, pp. 1036-1041, 1999. 

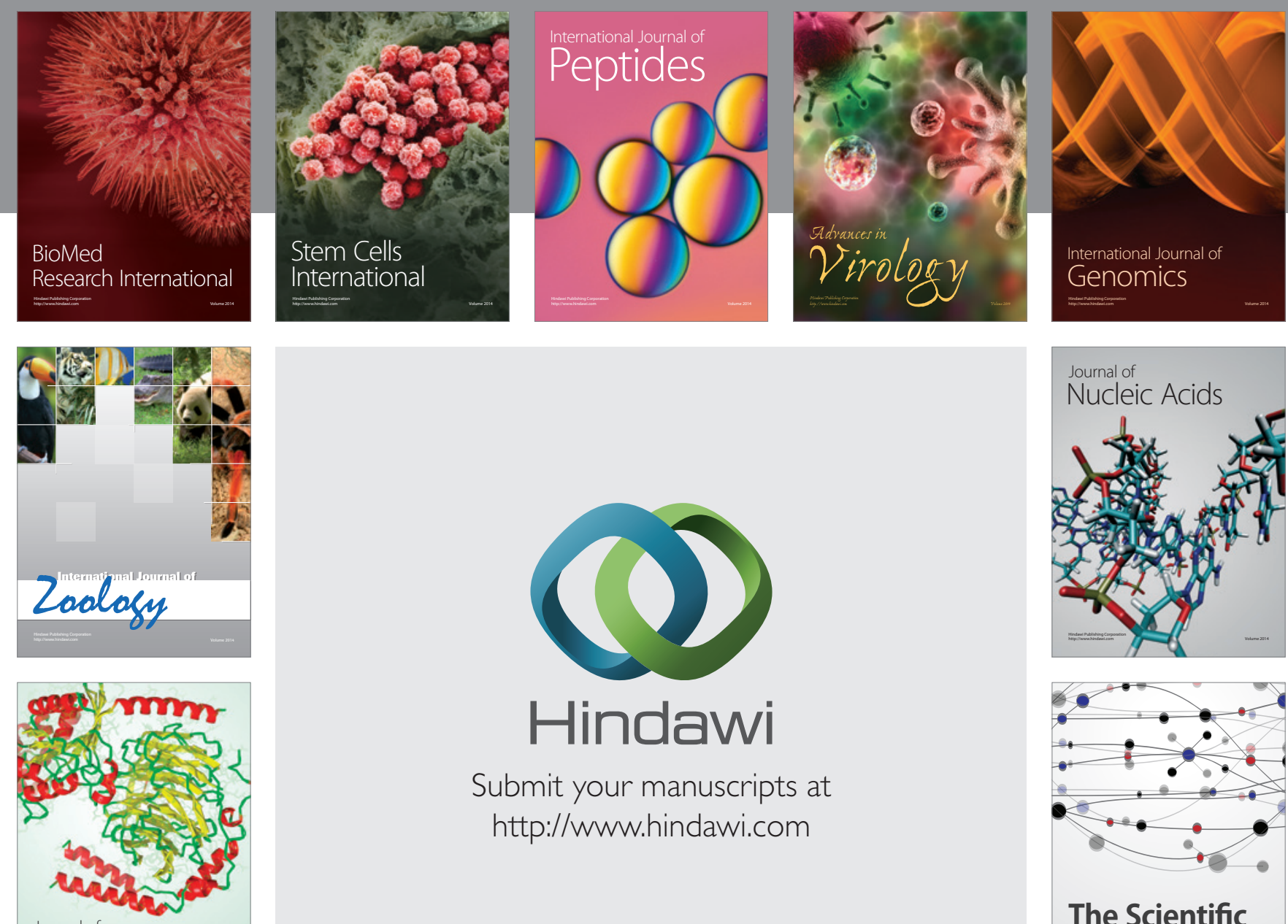

Submit your manuscripts at

http://www.hindawi.com

Journal of
Signal Transduction
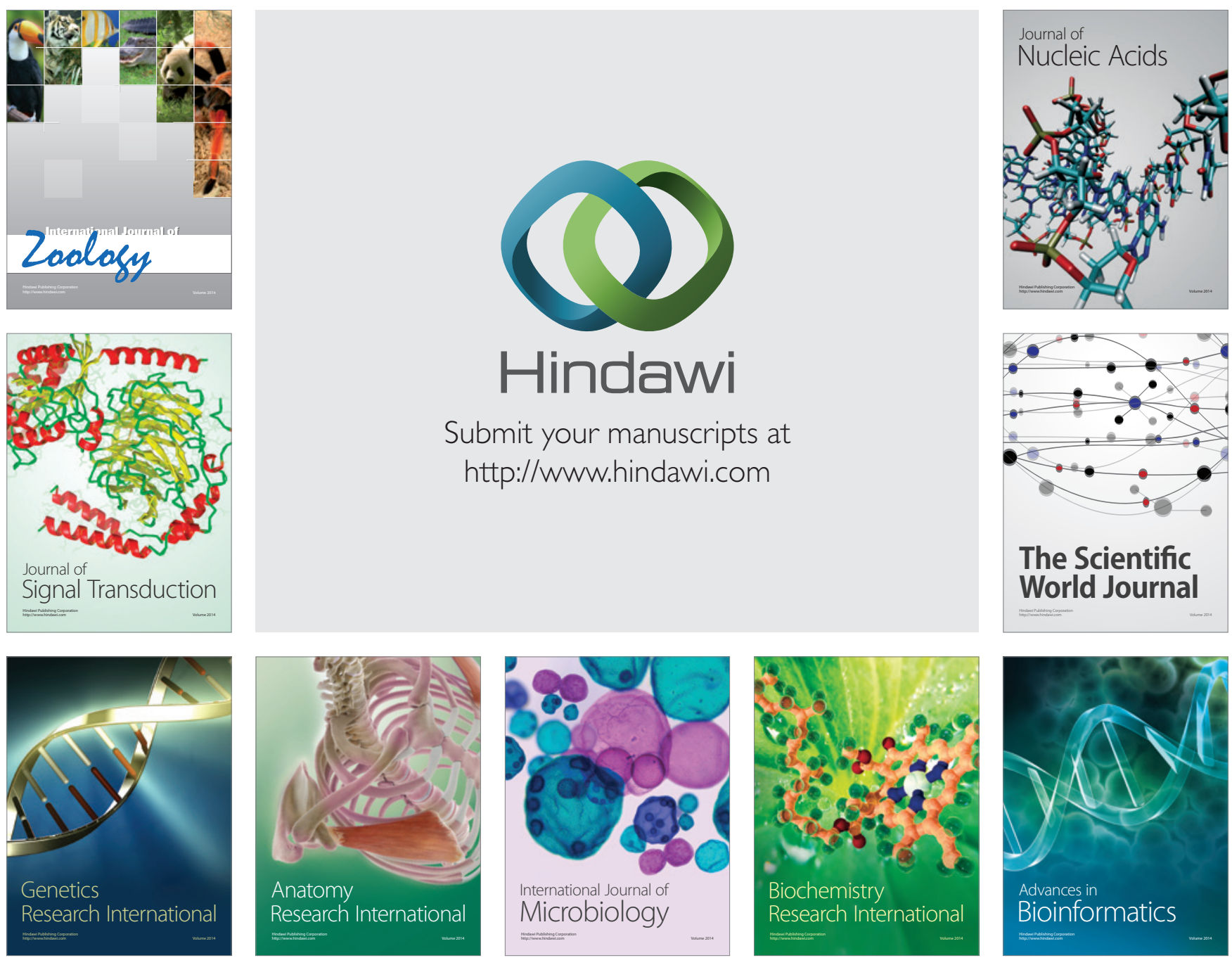

The Scientific World Journal
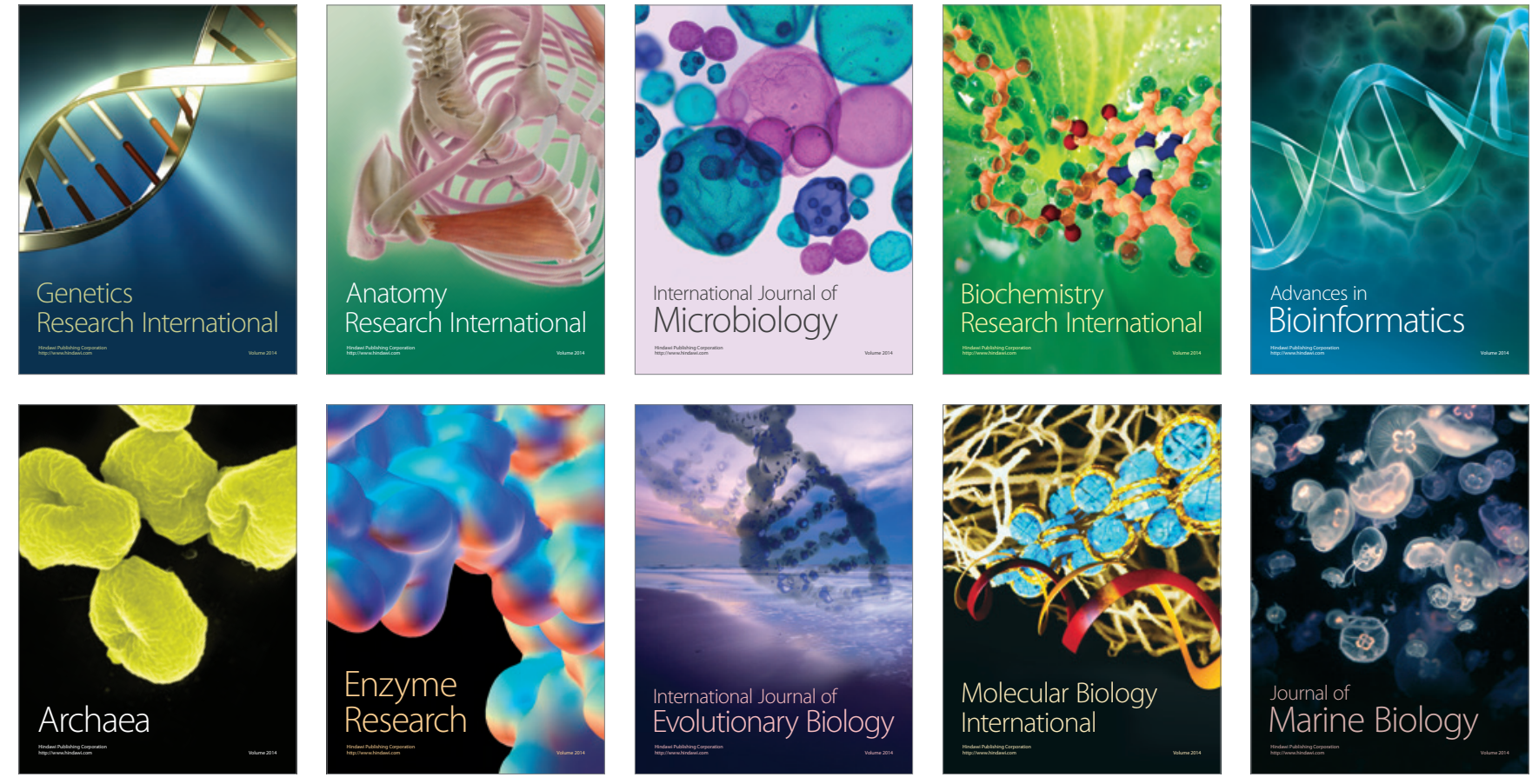